\title{
ARTICLE The RNA-binding protein tristetraprolin regulates RALDH2 expression by intestinal dendritic cells and controls local Treg homeostasis
}

Caroline La ${ }^{1,2}$, Bérengère de Toeuf ${ }^{2,3}$, Laure B. Bindels ${ }^{4}$, Laurye Van Maele ${ }^{1,2}$, Assiya Assabban ${ }^{1,2}$, Maxime Melchior ${ }^{1,2}$, Justine Smout ${ }^{1,2}$, Arnaud Köhler ${ }^{1,2}$, Muriel Nguyen ${ }^{1,2}$, Séverine Thomas ${ }^{1,2}$, Romuald Soin ${ }^{2,3}$, Nadège Delacourt ${ }^{2,3}$, Hsüehlei Li $^{2,3}$, Wenqian Hu$^{5}$, Perry J. Blackshear ${ }^{6}$, Véronique Kruys ${ }^{2,3}$, Cyril Gueydan ${ }^{2,3}$, Guillaume Oldenhove ${ }^{2,7}$ and Stanislas Goriely ${ }^{1,2}$

\begin{abstract}
AU-rich element (ARE)-mediated mRNA decay represents a key mechanism to avoid excessive production of inflammatory cytokines. Tristetraprolin (TTP, encoded by Zfp36) is a major ARE-binding protein, since $Z f f 36^{-1-}$ mice develop a complex multiorgan inflammatory syndrome that shares many features with spondyloarthritis. The role of TTP in intestinal homeostasis is not known. Herein, we show that $Z f p 36^{-/-}$mice do not develop any histological signs of gut pathology. However, they display a clear increase in intestinal inflammatory markers and discrete alterations in microbiota composition. Importantly, oral antibiotic treatment reduced both local and systemic joint and skin inflammation. We further show that absence of overt intestinal pathology is associated with local expansion of regulatory T cells. We demonstrate that this is related to increased vitamin A metabolism by gut dendritic cells, and identify RALDH2 as a direct target of TTP. In conclusion, these data bring insights into the interplay between microbiota-dependent gut and systemic inflammation during immune-mediated disorders, such as spondyloarthritis.
\end{abstract}

Mucosal Immunology (2021) 14:80-91; https://doi.org/10.1038/s41385-020-0302-x

\section{INTRODUCTION}

Epithelial barriers, such as gut and skin, are continuously exposed to environmental stimuli, including commensal and pathogenic microbes. In this context, it is essential for the host to control inflammatory processes in order to prevent local and systemic pathologies. The expression of key proinflammatory genes is regulated at the posttranscriptional level by the degradation of their mRNA. ${ }^{1}$ The presence of cis-acting AU-rich elements (AREs) in their $3^{\prime}$-untranslated regions ( $3^{\prime}$ UTR) allows for the recruitment of RNA-binding proteins that control their turnover and subcellular localization. ARE-mediated mRNA decay is critical to maintain immune homeostasis, since deletion of these regulatory elements in the Tnf gene is sufficient to trigger a spontaneous, microbiotadependent transmural inflammation in the distal ileum, as well as joint pathology. ${ }^{2-4}$ The major trans-acting regulatory factor in ARE-mediated Tnf mRNA decay is tristetraprolin (TTP), encoded by the Zfp36 gene. TTP targets ARE-containing mRNAs for degradation by recruitment of deadenylase complexes. ${ }^{5,6}$ In addition to TNF, TTP controls the expression of multiple inflammatory mediators, such as IL-23, IL-6, CXCL2, or GM-CSF. ${ }^{7-9}$ As a result, Zfp36 $^{-/-}$mice develop a TNF- and IL-23-dependent syndrome characterized by sero-negative arthritis, psoriatic-like skin lesions and systemic inflammation. ${ }^{10,11}$ Many of these features are reminiscent of spondyloarthritis ( $\mathrm{SpA})$, a group of interrelated immune-mediated inflammatory rheumatic conditions comprising ankylosing spondylitis (AS), psoriatic arthritis, arthritis/spondylitis with inflammatory bowel disease, and reactive arthritis. ${ }^{12,13}$ Multiple epidemiological studies have established a link between epithelial inflammation and SpA. ${ }^{12,14}$ Along these lines, we have previously shown that conditional deletion of TTP in epidermal cells was sufficient to trigger most of the cardinal features of the "TTP-deficiency" syndrome, including joint pathology. ${ }^{15}$ These observations suggest that TTP plays a major role in the maintenance of immune homeostasis at epithelial barriers. One could therefore expect that absence of TTP would lead to dysregulated production of inflammatory cytokines in response to gut microbiota. Herein, we show that, in contrast to $\operatorname{Tn} f^{\triangle \mathrm{ARE}}$ mice, deletion of Zfp36 is not accompanied by histological signs of gut pathology. Nevertheless, Zfp $36^{-1-}$ mice display a microbiota-dependent increase in intestinal inflammatory markers that corresponds with the onset of systemic, joint, and skin inflammation. We further show that lack of overt intestinal pathology is associated with local expansion of regulatory T cells (Tregs). We identified Aldh1a2 (encoding RALDH2) as a direct target of TTP in gut dendritic cells, linking increased retinoic acid (RA) metabolism and the local development and function of Tregs. Taken together, these data provide valuable insights into the role of

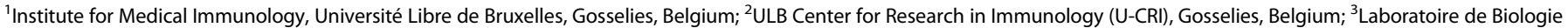

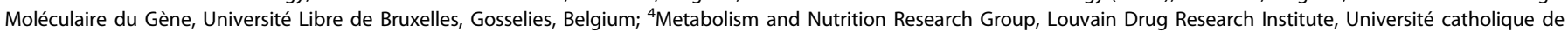

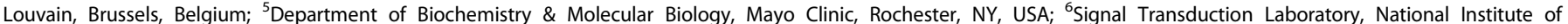
Environmental Health Sciences, Research Triangle Park, NC, USA and ${ }^{7}$ Laboratoire d'Immunobiologie, Université Libre de Bruxelles, Gosselies, Belgium

Correspondence: Stanislas Goriely (stanislas.goriely@ulb.be)

These authors contributed equally: Caroline La, Bérengère de Toeuf

These authors jointly supervised this work: Cyril Gueydan, Guillaume Oldenhove, Stanislas Goriely

Received: 20 December 2019 Revised: 10 April 2020 Accepted: 21 April 2020

Published online: 28 May 2020 
intestinal mucosal homeostasis during immune-mediated inflammatory disorders, such as SpA.

\section{RESULTS}

Zfp $36^{-1-}$ mice do not exhibit intestinal pathology despite increased inflammatory markers

Since ARE-mediated control of Tnf mRNA stability plays a major role in promoting gut immune homeostasis, ${ }^{2}$ we reasoned that this could be driven by TTP. For this purpose, we evaluated the histological features of the ileum and colon of 9-month-old $Z \mathrm{fp} 36^{-/-}$mice. At this age, all mice display clinical signs of systemic inflammation (reduced weight in comparison with their Zfp $36^{+/+}$or Zfp $36^{+/-}$littermates) and dactylitis. However, we did not observe major cellular infiltration, distortion of the crypts, atrophy or flattening of the villi in histological sections of the terminal ileum (Fig. 1a). Instead, crypt depth and villus length were even slightly greater. The levels of mRNA expression of a tight junction protein (Ocln, coding for Occludin), and markers of Paneth and goblet cells (Lyz1 and Muc2, respectively), were similar between both groups, suggesting that the ileal barrier architecture as well as epithelial subtypes are maintained in $Z f p 36^{-/-}$mice (Fig. 1 b). These results indicate that $Z f p 36^{-1-}$ mice do not display overt signs of pathology in the small intestine. We reached similar conclusions for the large intestine (Fig. 1c). In comparison, Tnf ${ }^{\triangle A R E}$ mice display clear signs of inflammation and villus destruction as early as 7 weeks of age. ${ }^{2}$ Despite this observation, fecal lipocalin-2 (LCN2), a classical marker of gut inflammation, was clearly increased in $Z \mathrm{fp} 36^{-1-}$ mice as compared with their littermates (Fig. 1d). Furthermore, FACS analysis revealed increased numbers of neutrophils in their lamina propria (SI LP) and mesenteric lymph nodes (MLN) (Fig. 1e). We also observed increased mRNA levels for known direct targets of TTP, such as Tnf, $C x C l 2, \| 11 b$, and II23a, along with $L c n 2$ and antimicrobial peptides, in the small intestine and to a lesser extent in the colon (Fig. 1f, g). Taken together, these results indicate that $Z f p 36^{-/-}$mice exhibit clear stigmata of local gut inflammation but do not develop overt ileitis nor colitis.

Microbiota-dependent subclinical gut inflammation contributes to the TTP-deficiency syndrome

To determine whether this subclinical gut inflammation was associated with alteration of the microbiome composition, we performed 16S rDNA sequencing of fecal extracts (collected from 5-month-old sex-matched $Z f p 36^{+/+}$and $Z f p 36^{-1-}$ littermates separated after weaning). There was no difference in total fecal bacteria levels in the two groups (Fig. 2a). We did not observe
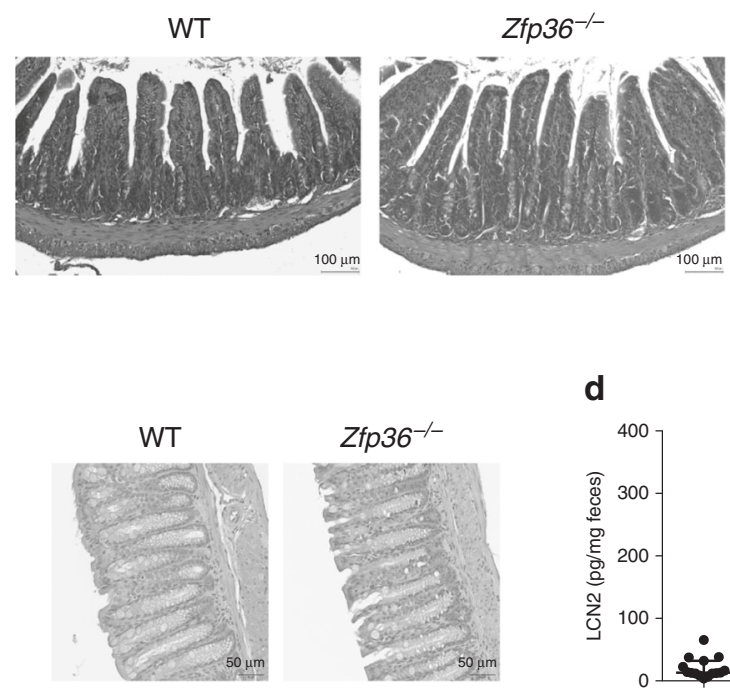

f

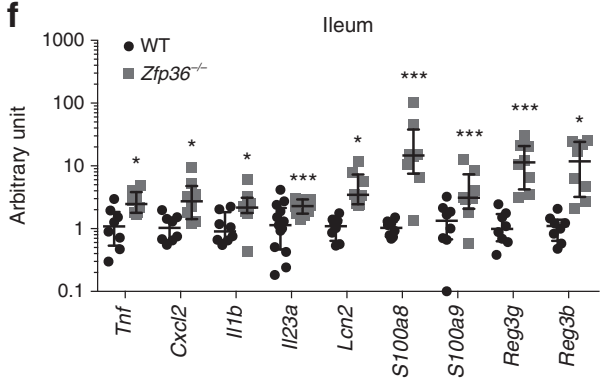

d

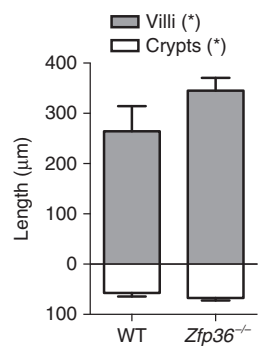

e b
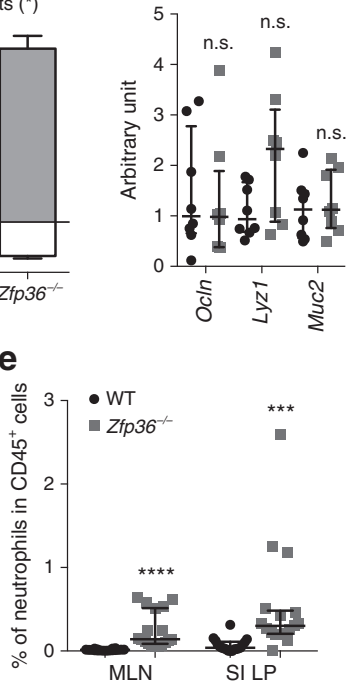

Colon

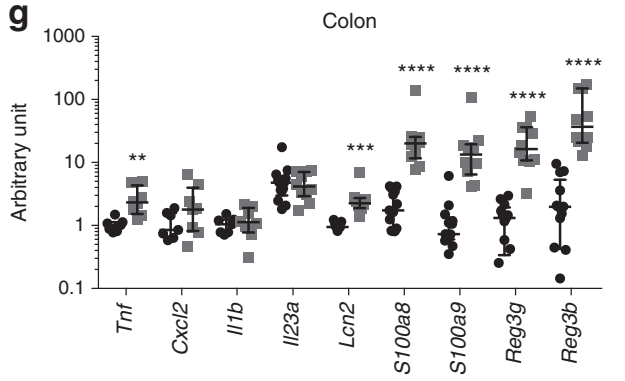

Fig. 1 Subclinical gut inflammation in Zfp $36^{-/-}$mice. a Villus length and crypt depth in ilea from mice of 6-9 months of age ( $n=5-8$ mice/ group, pooled from two independent experiments) with representative pictures of hematoxylin-eosin (HE) stained ilea. b Gene expression of a tight junction protein (Ocln, coding for Occludin), and markers of Paneth and goblet cells (Lyz1 and Muc2, respectively) by RTqPCR ( $n=8$ mice/ group, pooled from two independent experiments). c Representative pictures of HE stained colons. d Fecal lipocalin-2 (LCN2) by ELISA ( $n=$ 14-16 mice/group, pooled from two independent experiments). e Neutrophil infiltration of the mesenteric lymph nodes (MLN) and the small intestinal lamina propria (SI LP) by flow cytometry ( $n=18$ mice/group, pooled from six independent experiments). Cytokine and antimicrobial peptide gene expression in total ileum (f) and colon (g) by RTqPCR ( $n=8$ mice/group, pooled from two independent experiments). Results in $\mathbf{b}$, $\mathbf{f}$ and $\mathbf{g}$ are expressed in arbitrary units (normalized against $A c t b$ mRNA levels, and each result expressed relative to its WT control group). Results are given as median \pm interquartile range, and each dot represents a single mouse. Statistical significance (ns nonsignificant, ${ }^{*} P<0.05,{ }^{* *} P<0.01$, ${ }^{* * *} P<0.001$, $\left.{ }^{* * *} P<0.0001\right)$ was assessed by the Mann-Whitney test $(\mathbf{a}, \mathbf{b}, \mathbf{d}, \mathbf{f}, \mathbf{g})$ or the Kruskal-Wallis test $(\mathbf{e})$. 


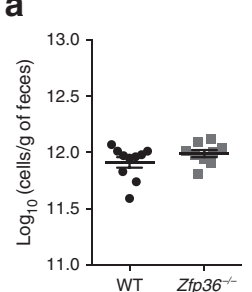

b

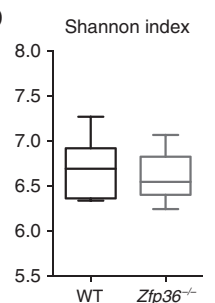

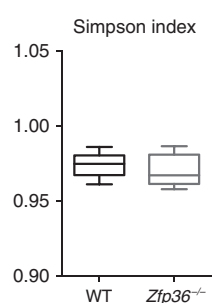

d

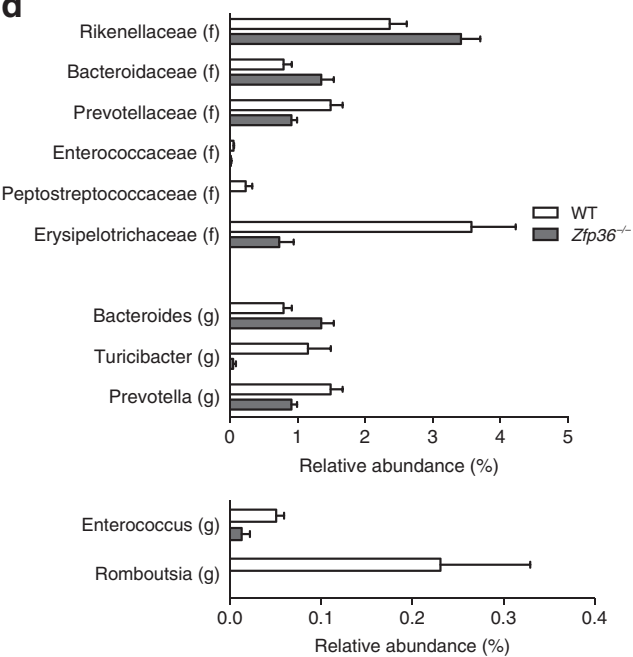

C

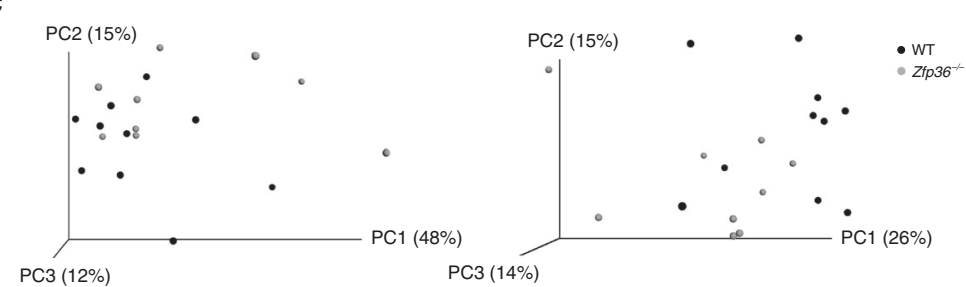

g

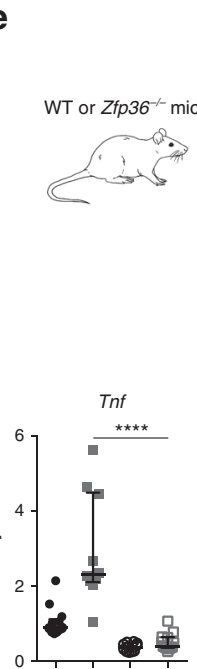

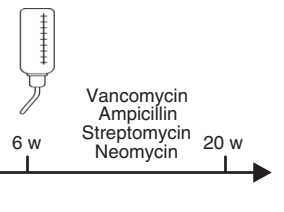

- WT control - Zfp $36^{-1-}$ control Zfp $36^{- \text {contro }}$ 口 $Z$ fp $36^{--}+\mathrm{ABT}$ f

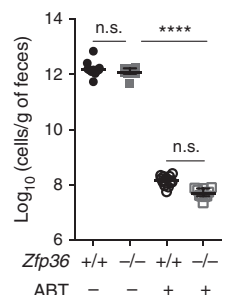

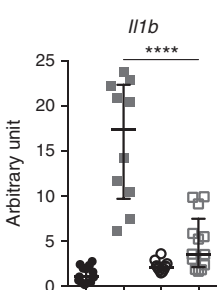
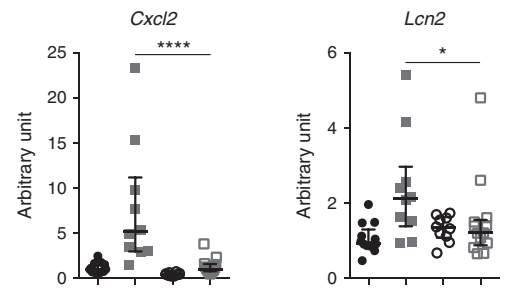

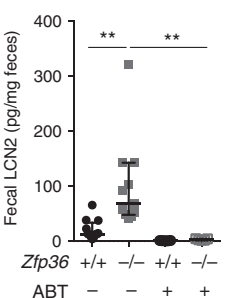

h

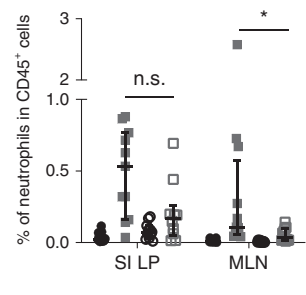

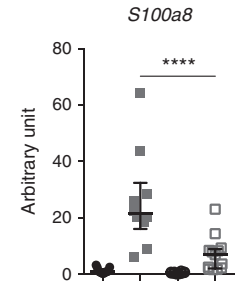
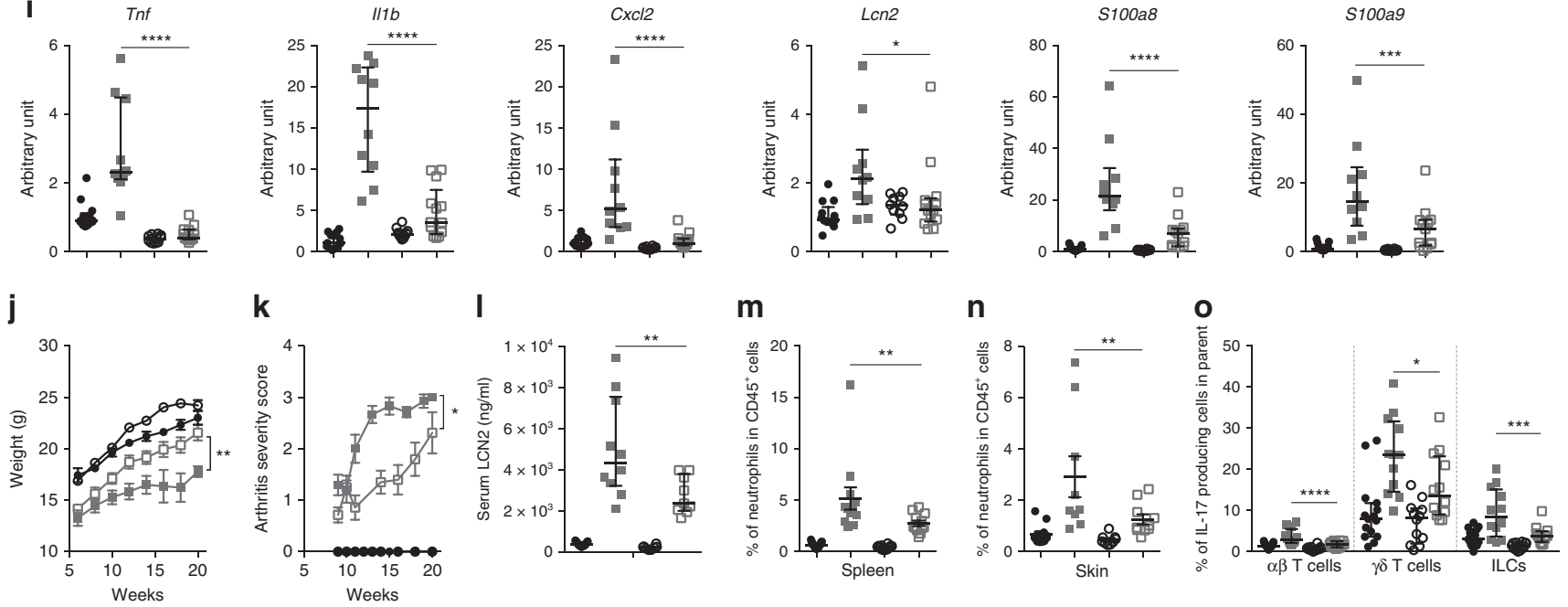

Fig. 2 Contribution of the gut microbiota to local and systemic inflammation in $\mathbf{Z f p} 3 \mathbf{6}^{-/-}$mice. a-d Feces were collected from WT or $Z f p 36^{-1-}$ mice at 5 months of age for bacterial DNA sequencing. a Total fecal bacteria levels by absolute quantitative RTqPCR $(n=4-10$ mice/ group). b Shannon and Simpson alpha-diversity indexes. c Principal coordinates analyses of the Morisita-Horn and Bray-Curtis beta-diversity metrics. In these analyses, genotype explains $13 \%$ and $23 \%$, respectively, of the dataset variance (ANOSIM analyses, 1000 permutations, $p<$ 0.05). d Families and genera significantly affected in $Z f p 36^{-1-}$ mice $(q$ value $<0.1)$. e Modulation of gut microbiota by broad-spectrum antibiotic therapy (ABT) in Zfp36 ${ }^{-/-}$mice and WT controls, from 6 weeks of age until the day of sacrifice at 20 weeks of age, followed by flow cytometry, ELISA, and RTqPCR experiments. $f$ Total fecal bacteria levels by absolute quantitative RTqPCR in feces collected 2 months after the start of ABT ( $n=8-12$ mice/group, pooled from two independent experiments). $\mathbf{g}$ Lipocalin-2 levels by ELISA in feces collected before ( $n=$ 11-13 mice/group, pooled from two independent experiments) and 2 months after the start of ABT ( $n=5-7$ mice/group). h Neutrophil infiltration of the small intestinal lamina propria (SI LP) and mesenteric lymph nodes (MLN) by flow cytometry $(n=12-17$ mice/group, pooled from three independent experiments). i Cytokine and antimicrobial peptide gene expression in total ileum by RTqPCR, expressed in arbitrary units normalized against Actb mRNA levels and relative to the WT control group ( $n=10-13$ mice/group, pooled from two independent experiments). $\mathbf{j}$ Weight and $\mathbf{k}$ arthritis severity follow-up ( $n=12-17$ mice/group, pooled from three independent experiments). (I) Serum levels of lipocalin-2 by ELISA ( $n=8-13$, pooled from two independent experiments). $\mathbf{m}$ Neutrophil infiltration of the spleen ( $n=12-17$ mice/group, pooled from three independent experiments) and $\mathbf{n}$ the skin ( $n=9-12$ mice/group, pooled from two independent experiments) by flow cytometry. o IL-17 producing cells of the skin by flow cytometry $(n=12-15$ mice/group, pooled from three independent experiments). Results are given as median \pm interquartile range and each dot represents a single mouse. Statistical significance (ns nonsignificant, ${ }^{*} P<0.05$, ${ }^{* *} P<$ $\left.0.01,{ }^{* * *} P<0.001,{ }^{* * *} P<0.0001\right)$ was assessed by two-way ANOVA test $(\mathbf{f}-\mathbf{i}, \mathbf{I}-\mathbf{o})$. 
significant differences in alpha diversity between the two groups based on Shannon and Simpson indexes. With principal coordinates analyses of the Morisita-Horn and Bray-Curtis beta-diversity metrics, genotype only explained $13 \%$ and $23 \%$, respectively, of the dataset variance (Fig. 2b, c). Detailed analysis of the 16S rDNA sequencing data revealed the presence of 56 taxa. Out of these, the relative abundance of 11 taxa were found to be significantly altered in $Z f p 36^{-1-}$ mice (corrected $p$ value $<0.1$, Fig. $2 d$ ). In particular, Rikenellaceae and Bacteroidaceae families that were found to be enriched in the terminal ileum of AS patients ${ }^{16}$ were significantly increased in $Z f p 36^{-1-}$ mice; furthermore, Prevotellaceae, depleted in these patients, were also decreased in these mice (Fig. 2d). Several of the amplicon sequencing variants (ASVs) that were increased in $Z f p 36^{-1-}$ mice were identified as Porphyromonadaceae members, a family that was also found to be increased in AS patients (Supplementary Table S1). Thus, TTPdeficient mice display a discrete microbial signature that shares several features with the ones previously described in AS patients.

Next, we investigated the potential impact of the gut microbiota on the local and systemic inflammation developed by $Z f p 36^{-1-}$ mice. For this purpose, we modulated the microbiota by broad-spectrum antibiotics in the drinking water, beginning at 6 weeks of age (Vancomycin $0.5 \mathrm{~g} / \mathrm{l}$, Ampicillin $1 \mathrm{~g} / \mathrm{l}$, Streptomycin $1 \mathrm{~g} / \mathrm{l}$, and Neomycin $1 \mathrm{~g} / \mathrm{l}$ ). We maintained this treatment until analysis at 20 weeks of age (Fig. 2e). As expected, antibiotic treatment clearly decreased the number of total fecal bacteria in both groups (Fig. 2f). Local gut inflammation was reduced, with normalization of fecal LCN2 levels (Fig. $2 \mathrm{~g}$ ) and decreased mRNA expression of ileal and colonic inflammatory markers in treated vs control $Z \mathrm{fp} 36^{-1-}$ mice (Fig. $2 \mathrm{i}$ and Supplementary Fig S1). There was also a trend towards reduced neutrophil infiltration in the $\mathrm{SI}$ LP and MLN from Zfp $36^{-/-}$mice (Fig. 2h). In order to further define the contribution of microbial recognition, signaling through IL-1 receptor family and inflammasome pathways to gut inflammation, we deleted Zfp36 in mice of $M y d 88^{-1-}$ and Casp1/ $11^{-/-}$backgrounds. Both MyD88 and Caspase 1 have been found to be critical for intestinal inflammation in various models. ${ }^{4,17}$ We assessed mRNA expression of cytokines and inflammatory markers in ileum samples collected between 5 and 7 months of age (Supplementary Fig S2). We observed that Tnf upregulation in the absence of TTP was MyD88- and Caspase 1/11-dependent. This was not the case for $111 b$ and $C x C / 2$. Induction of $L c n 2, S 100 a 8$, and S100a9 was strongly MyD88- and to a lesser extent Caspase 1/11dependent. These results support the role of microbiota-derived signals and possibly of IL-1 family-related signaling pathways as a trigger for local gut inflammation in $Z f p 36^{-1-}$ mice.

In order to evaluate whether the altered gut microbiota from $Z f p 36^{-1-}$ mice had an effect on intestinal inflammation when transferred to WT mice, we analyzed gut inflammatory mediators in littermates directly separated after weaning or cohoused for several months with $Z \mathrm{fp} 36^{-1-}$ mice. WT mice that were co-housed displayed slight but significant increase in the expression of $I / 1 b$, Lcn2, Reg $3 b$, and Reg $3 g$ in comparison with separated littermates (Supplementary Fig S3). This result suggests that altered gut microbiota from $\mathrm{Zfp} 6^{-1-}$ mice contributes to gut inflammation, although this effect did not appear to be dominant.

We then evaluated the effect of oral antibiotic treatment on systemic, joint and skin features of the TTP-deficiency syndrome. Strikingly, Zfp $36^{-/-}$mice on antibiotics showed improved weight gain over time, and a prominent delay in the development of the joint pathology, as compared with untreated $Z \mathrm{fp} 36^{-/-}$mice (Fig. 2j, k). Likewise, markers of systemic inflammation such as serum LCN2 levels and accumulation of neutrophils in the spleen were significantly decreased (Fig. 2l, m). We previously demonstrated that TTP-deficient mice developed an IL-17-dependent skin inflammation. ${ }^{11}$ Upon oral antibiotics treatment, we observed reduced neutrophil recruitment to the skin, as well as decreased frequency of $\mathrm{IL}-17$ production by lymphoid cells, including aßT cells, ILCs and $\gamma \delta T$ cells (Fig. $2 n, 0$ ). These results strongly suggest that microbiota-dependent intestinal inflammation contributes to the systemic, joint and skin pathologies developed by Zfp $36^{-1-}$ mice.

Expansion of Tregs in the small intestine of $Z f p 36^{-/-}$mice Although $Z \mathrm{fp} 36^{-1-}$ mice display clear microbiota-dependent disruption of intestinal immune homeostasis that contributes to systemic inflammation, this process does not lead to overt gut pathology. We explored the mechanisms that could account for this apparent paradox. The TTP-deficiency syndrome is associated with dysregulated Th17 responses. ${ }^{11}$ We evaluated the frequency of Th17 cells in the SI LP of $Z f p 36^{-/-}$mice and littermates by flow cytometry. While the proportion and absolute number of RORyt ${ }^{+}$ cells among conventional Th cells was comparable in both groups (Fig. 3a), their capacity to produce IL-17 (Fig. 3b) along with steady-state $1117 a$ mRNA levels in ileal samples (Fig. 3c) were significantly lower in $Z f p 36^{-/}$mice, suggesting that Th17 function rather than differentiation and proliferation was impaired. We therefore hypothesized that local regulatory mechanisms must be at play. We observed 1.5- and 2-fold increases in the proportion of Foxp $3^{+}$Tregs in $\mathrm{CD}^{+}{ }^{+} \mathrm{T}$ cells from the MLN and SI LP of Zfp $36^{-1-}$ mice respectively, along with an increase in the absolute number for SI LP Tregs (Fig. 3d, e). Of note, this was not the case in the spleen and in the LP isolated from the colon, suggesting that gut micro-environmental factors associated to the small intestine are responsible for this observation. We noted that the expansion of SI LP Tregs in $Z \mathrm{fp} 36^{-/-}$mice was dependent on microbiota-derived factors as we observed decreased frequency in ABT-treated animals (Supplementary Fig. 4). Importantly, SI LP Tregs from Zfp $36^{-/-}$ mice displayed an increased capacity to produce IL-10, and we also noted increased $/ / 10$ mRNA levels in ileal samples under steady-state conditions (Fig. $3 \mathrm{f}, \mathrm{g}$ ). It is possible to discriminate several subsets of Tregs based on their expression of transcription factors. ${ }^{18}$ We observed a relative decrease in the proportions of thymic-derived GATA $^{+}$and microbiota-dependent RORyt ${ }^{+}$ subsets, as the expansion of Foxp3 $3^{+}$Tregs in $Z \mathrm{fp} 36^{-/-}$mice was restricted to GATA $3^{-}$RORyt $^{-}$cells (Fig. 3h). Taken together, these results indicate that both the frequency and the functionality of Tregs are enhanced in the small intestine of $\mathrm{Zfp} 36^{-/-}$mice. Furthermore, the pattern of transcription factors they express suggests that they are induced in the periphery in response to food-borne antigens. ${ }^{18}$

Dendritic cells are the main drivers of this enhanced Treg development and function in the SI LP of $Z \mathrm{fp} 36^{-/-}$mice In order to decipher the mechanisms involved in this expansion of Tregs, we evaluated the expression of TTP in the different cellular compartments of the small intestine under steady-state conditions. As most anti-TTP antibodies cannot be reliably used for FACS staining, we used a newly developed Zfp36-V5 epitope tagged knock-in mouse generated by CRISPR/Cas9-mediated genome editing. ${ }^{19}$ We observed consistent TTP-V5 staining in SI LP myeloid cells, in particular conventional dendritic cells (CDC) and monocytes, and to a lesser extent in macrophages and neutrophils (Fig. 4a). We also noted the expression of TTP-V5 in up to $30 \%$ of Epcam ${ }^{+}$epithelial cells. In contrast, expression of TTP in most SI LP and intraepithelial lymphoid subsets, including Tregs, was low or undetectable. We observed higher expression of TTPV5 in CDC1 isolated from the SI LP in comparison with their counterparts from the spleen or the lung (Fig. 4b). We reached similar conclusions for $\mathrm{Ly}_{6 \mathrm{C}} \mathrm{C}^{+}$monocytes. These results indicate that DCs and monocytic cells from the gut express high levels of TTP under physiological conditions. To define whether the expression of TTP in myeloid cells isolated from the MLN and SI LP was dependent on the presence of gut microbiota, Zfp36-V5 
a

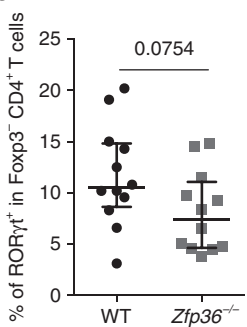

C

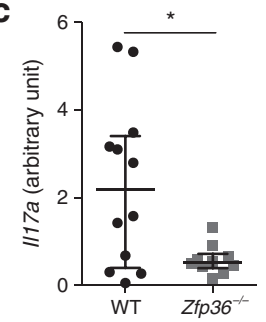

e

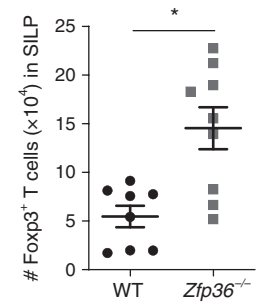

g

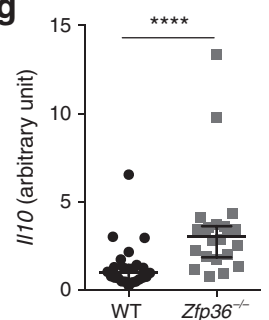

b

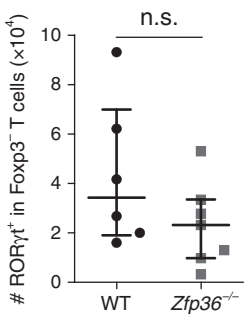

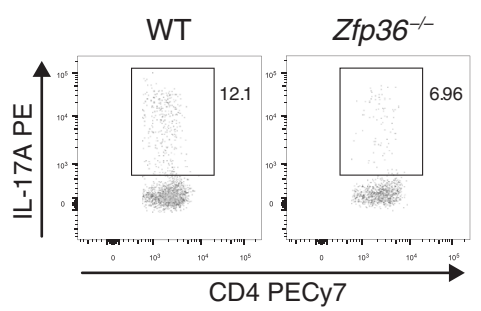

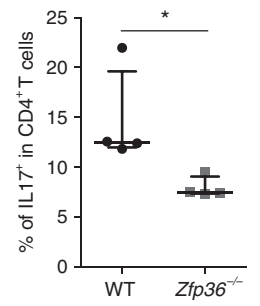

d

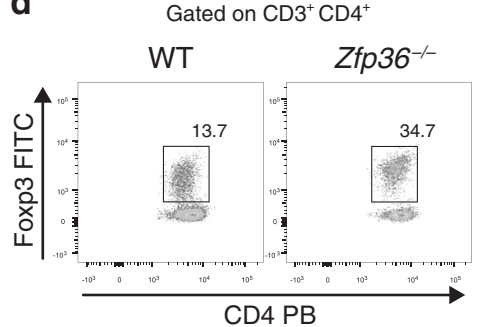

f

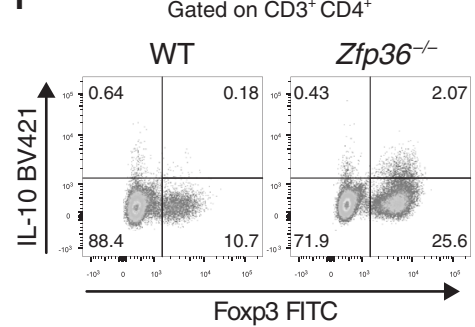

h

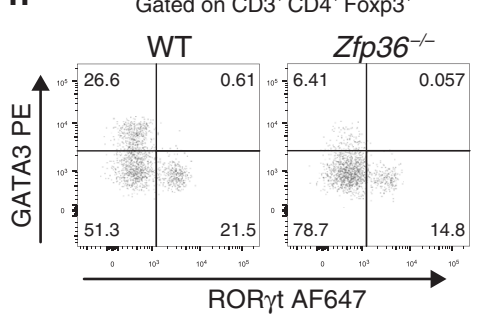

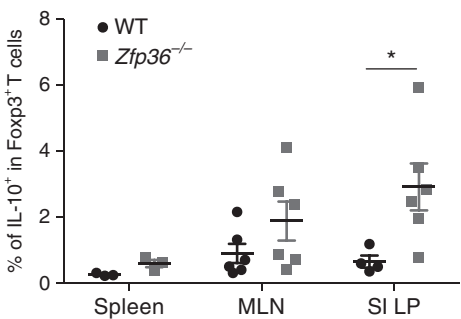
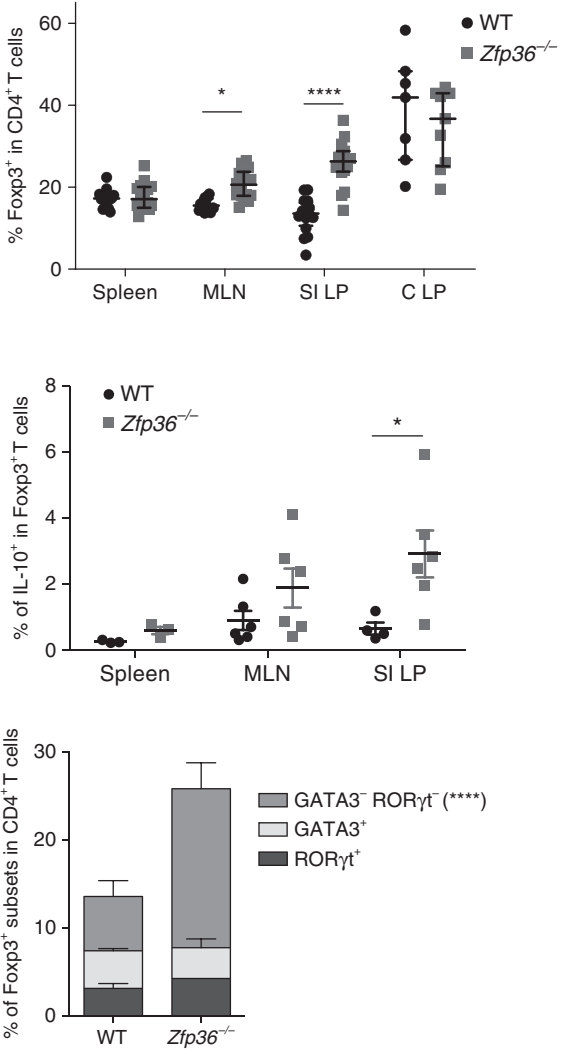

Fig. 3 Increased proportion of Tregs in the small intestinal GALT of Zfp36 ${ }^{-1-}$ mice. Flow cytometry and RTqPCR results in WT or Zfp36 ${ }^{-1-}$ mice. a Proportion (left) of Th17 cells (ROR $\gamma \mathrm{t}^{+}$cells) among Foxp3 $3^{-}$CD4 T cells in SI LP ( $n=12$ mice/group, pooled from three independent experiments) and absolute number of Th17 in SI LP (right) ( $n=6-7$ mice/group, pooled from two independent experiments). $\mathbf{b}$ Proportion of IL-17 producers among total CD4 T cells ( $n=4$ mice/group). c $/ 117 a$ gene expression in total ileum $(n=10-12$ mice/group, pooled from three independent experiments). d Proportion of Tregs (Foxp3 ${ }^{+}$cells) among CD4 T cells in spleen, MLN, SI LP ( $n=16$ mice/group, pooled from five independent experiments), and colon lamina propria (C LP) ( $n=7-9$ mice/group, pooled from three independent experiments). e Absolute number of Tregs (Foxp3 ${ }^{+} \mathrm{CD}^{+}$T cells) in SI LP $(n=8-9$ mice/group, pooled from three independent experiments). $\mathbf{f}$ Proportion of IL-10 producing Foxp $3^{+}$Treg in spleen, MLN, and SI LP ( $n=4-6$ mice/group, pooled from two independent experiments). $g / 110$ gene expression in total ileum $\left(n=23\right.$ mice/group, pooled from five independent experiments). h Proportion of Foxp3 ${ }^{+}$subsets (GATA3 ${ }^{+}$, ROR $\gamma \mathrm{t}^{+}$and GATA3 ${ }^{-}$ROR $^{-} \mathrm{t}^{-}$cells) among CD4 T cells ( $n=7-8$ mice/group, pooled from two independent experiments). RTqPCR results are expressed in arbitrary units, normalized against Actb mRNA levels and relative to the WT group. Results are given as median \pm interquartile range and each dot represents a single mouse. Statistical significance (ns nonsignificant, ${ }^{*} P<0.05,{ }^{* *} P<0.01,{ }^{* *} P<0.001,{ }^{* * *} P<0.0001$ ) was assessed by Mann-Whitney test $(\mathbf{a}-\mathbf{c}, \mathbf{e}, \mathbf{g})$ or two-way ANOVA test $(\mathbf{d}, \mathbf{f}, \mathbf{h})$.

mice were treated with broad-spectrum antibiotics in the drinking water during 4 weeks (Supplementary Fig S5). Antibiotic treatment reduced the frequency of monocytes and neutrophils in the MLN but not in the SI LP. The percentage and intensity of TTP-V5 expression among $\mathrm{CD}_{103^{+}}$and double-positive DCs subsets from the MLN but not the SI LP were decreased in these conditions. These observations suggest that TTP expression in DCs that migrate from the gut to the MLN is partially dependent on microbiota-derived signals. Based on these results, we decided to evaluate the role of TTP within CD11c-expressing cells (CD11c-Cre$Z f p 36^{\mathrm{fl} / f l} ; Z f p 36^{\Delta D C}$, targeting dendritic cells but also macrophage subsets) and myeloid cells (LysM-Cre-Zfp $36^{\mathrm{fl} / \mathrm{fl}} ; Z_{\mathrm{fp}} 36^{\Delta M}$ mice, targeting macrophages, neutrophils, and monocytes). As previously described, neither strain developed signs of systemic inflammation. ${ }^{7,15,20}$ Furthermore, mRNA expression of inflammatory cytokines and antimicrobial peptides was not increased in ileal samples (Fig S6). In comparison with $Z f p 36^{f / f l}$ controls, we observed an expansion of SI LP Tregs in Zfp $36^{\Delta D C}$ but not in $Z f p 36^{\Delta M}$ mice, suggesting that TTP deficiency in DCs rather than in other myeloid subsets affects Treg homeostasis (Fig. 4c). However, we cannot exclude that this effect was also mediated by $\mathrm{CD} 11 \mathrm{c}^{+}$ intestinal macrophages. As in $Z f p 36^{-1-}$ mice, the proportion of 
a
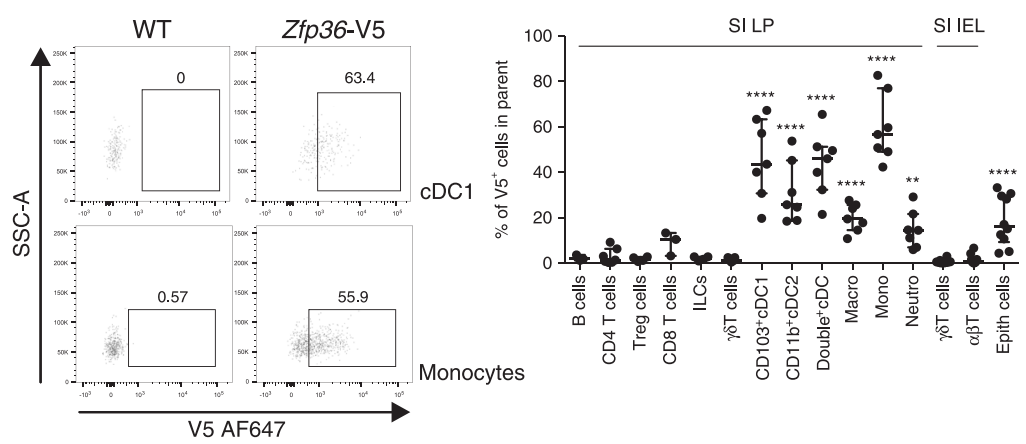

b
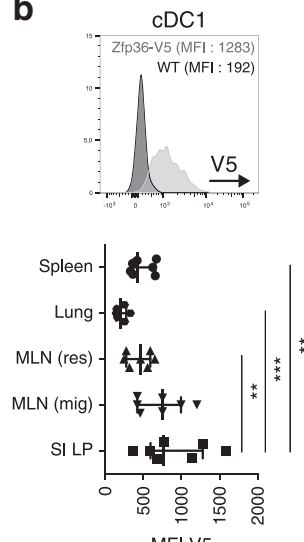

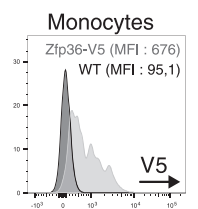

Spleen -1

Lung-

MLN

SI LP - *

- \&

MFI V5

C

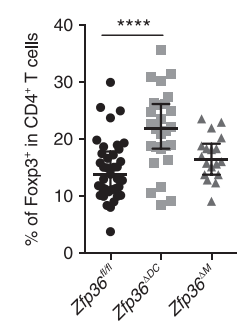

f

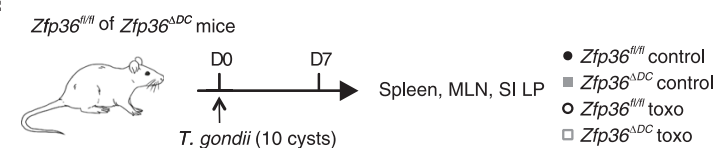

d

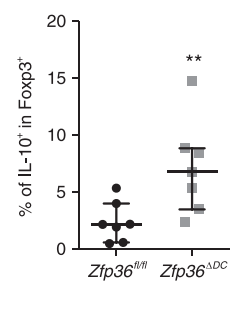

$\square$ Zfp36 ${ }^{\Delta D C}$ toxo e OTII Foxp3-GFP mice Zfp $36^{1 / 11}$ or Zfp $36^{\Delta 0 C}$ mice

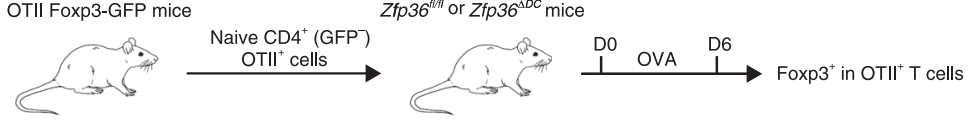

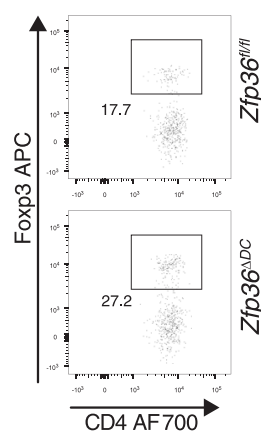
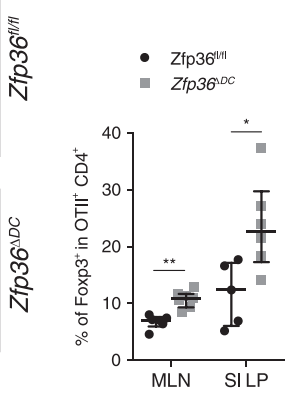

g
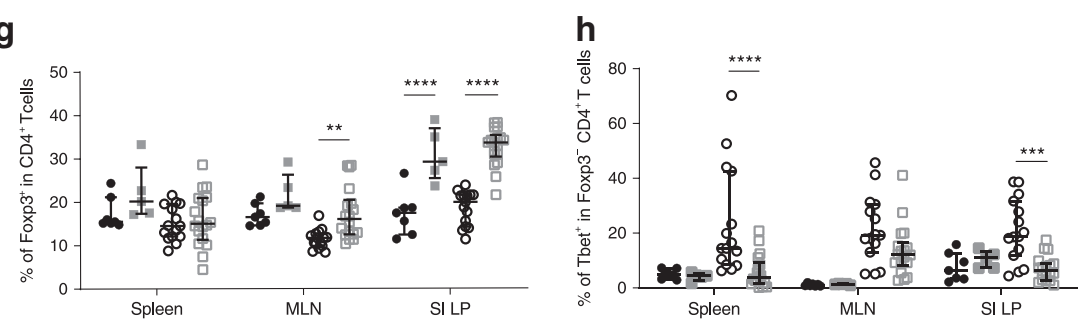

Fig. 4 Enhanced SI LP Treg development and function upon Zfp36 deletion in dendritic cells. a, b Flow cytometry results in WT or Zfp36-V5 epitope tagged knock-in mice, using an anti-V5 antibody. a Proportion of V $5^{+}$cells in different immune cell populations $(n=3-7$ mice/group, pooled from three independent experiments) and epithelial cells $(n=10$ mice/group, pooled from three independent experiments) in the small intestine $(\mathrm{SI})$ at steady state. b Mean fluorescence intensity of V5 in conventional dendritic cells type 1 (cDC1: $\mathrm{MHCII}^{+} \mathrm{CD} 11 \mathrm{c}^{+} \mathrm{CD} 103^{+} \mathrm{CD} 11 \mathrm{~b}^{-}$cells) and monocytes $\left(\mathrm{CD} 11 \mathrm{~b}^{+} \mathrm{Ly}_{6 \mathrm{C}^{+}} \mathrm{Ly}_{6 \mathrm{G}}{ }^{-}\right.$) from lymphoid organs (spleen, mesenteric lymph nodes) or barrier organs (SI LP, lung) ( $n=6-7$ mice/group from two independent experiments). In MLN, CDCs were further differentiated into migratory (mig) or residential (res) subtypes. c-h Flow cytometry results in conditional Zfp36 knock-out mice strains (CD11c-Cre Zfp36 $6^{f / f}$ : Zfp $36^{\Delta D C}$ and LysM-Cre Zfp36 $6^{f / f l}: Z f p 36^{\Delta M}$ ) and their control Zfp36 flox/flox (fl/fl) mice. c Proportion of Tregs (Foxp3 ${ }^{+}$cells) among CD4 T cells in SI LP ( $n=18-37$ mice/group, pooled from 3-5 independent experiments). d Proportion of IL-10 producing Foxp ${ }^{+}$Tregs in SI LP ( $n=7$ mice/ group, pooled from three independent experiments). e Increased peripheral Treg differentiation in SI LP from Zfp36 ${ }^{\triangle D C}$ mice in an oral tolerance model: $Z f p 36^{f / f / l}$ or $Z f p 36^{\Delta D C}$ mice were fed for 6 days with ovalbumin, after transfer of naive CD4 ${ }^{+} \mathrm{OTII}^{+}$cells sorted from OTII mice $(n$ =5-7 mice/group, pooled from two independent experiments). $\mathbf{f} Z \mathrm{fp} 36^{\mathrm{f} / \mathrm{fl}}$ and $Z \mathrm{fp} 36^{\Delta D C}$ mice were infected with ten cysts of $T$. gondii and sacrificed 7 days later to proceed to flow cytometry and RTqPCR experiments $(\mathbf{g}, \mathbf{h})(n=5-7$ control mice or $16-18$ infected mice, from three independent experiments). g Proportion of Tregs (Foxp3 ${ }^{+}$cells) among CD4 T cells in spleen, MLN, and SI LP. $\mathbf{h}$ Proportion of Tbet ${ }^{+}$cells among Foxp $3^{-}$CD4 T cells in spleen, MLN, and SI LP. Results are given as median \pm interquartile range and each dot represents a single mouse. Statistical significance $\left({ }^{*} P<0.05\right.$, ${ }^{* *} P<0.01$, ${ }^{* * *} P<0.001$, $\left.{ }^{* * * *} P<0,0001\right)$ was assessed by two-way ANOVA (a: WT vs. Zfp36-V5 tagged mice, b: MFI in SI LP from Zfp36-V5 tagged mice compared with MFI in other organs, $\mathbf{g}, \mathbf{h}$ ), by Kruskal-Wallis (c) or by Mann-Whitney test (d, e).

IL-10-producing cells among Tregs was also increased in Zfp36 ${ }^{\Delta \mathrm{DC}}$ mice as compared with Zfp36 f/f/I mice (Fig. 4d). One of the key roles of GATA ${ }^{-}{ }^{-}$RORYt ${ }^{-}$intestinal Tregs is the maintenance of oral tolerance to food-borne antigens. ${ }^{18}$ We therefore asked whether the deficiency of TTP in DCs affects de novo generation of intestinal Tregs upon oral administration of ovalbumin (OVA).
Naive Foxp3 ${ }^{-} \mathrm{CD}^{+} \mathrm{T}$ cells from transgenic OTII mice were adoptively transferred to Zfp $36^{f / / f}$ or Zfp $36^{\Delta \mathrm{DC}}$ mice. Mice were subsequently fed with OVA. We observed that conversion and expansion of OVA-specific Tregs in the small intestine and the MLNs was enhanced in $Z f p 36^{\Delta \mathrm{DC}}$ mice compared with $Z f p 36^{f / f l}$ mice (Fig. 4e). 
To further define whether Tregs from $Z f p 36^{\Delta D C}$ mice were efficient in the suppression of inflammation in vivo, we infected these mice orally with cysts of $T$. gondii (Fig. $4 \mathrm{f}$ ). In this model, Tregs are required to suppress excessive mucosal Th1 responses. ${ }^{21}$ At the peak of the infection, the proportion of Foxp $3^{+}$Treg cells among $\mathrm{CD}_{4}^{+}{ }^{+}$cells of the SI LP (and MLNs) remained increased in infected $Z f p 36^{\Delta \mathrm{DC}}$ mice compared with $Z f p 36^{\mathrm{fl} / \mathrm{fl}}$ infected mice, to the same extent as in noninfected mice (Fig. $4 \mathrm{~g}$ ). This increase in Treg cells was associated with a reduction of Tbet-expressing Th cells both locally and in the spleen (Fig. 4h). Taken together, our results strongly support the notion that enhanced Treg development and function in the small intestine occurs as a consequence of Zfp36 deletion in gut-associated DCs, and potentially controls the intestinal inflammation observed upon deletion of Zfp36.

\section{RALDH2 is a direct target of TTP}

Small intestine $\mathrm{CD}_{103^{+}} \mathrm{DCs}$ display strong tolerogenic properties and promote the conversion of naive CD4 T cells into Tregs through the production of RA. ${ }^{22}$ We therefore evaluated the role of TTP in DC homeostasis. There were no significant differences between $Z f p 36^{\Delta D C}$ and $Z f p 36^{\mathrm{f} / f l}$ mice in terms of either total SI LP DC frequency or in the proportions of $\mathrm{CD}_{103^{+}}, \mathrm{CD} 11 \mathrm{~b}^{+}$, and double-positive subsets (Fig. 5a, b). However, we observed a strong increase in retinaldehyde dehydrogenase (RALDH) activity in SI LP DCs of $Z f p 36^{\triangle D C}$ mice using Aldefluor (an RALDH fluorometric substrate) (Fig. $5 \mathrm{c}$ ). RALDH produces RA from retinaldehyde, a derivative of Vitamin $A$. In order to test if the increased capacity to generate RA by DCs was responsible for the expansion of Tregs in the gut of $Z f p 36^{\Delta D C}$ mice, we subjected these mice to a vitamin A-deficient (VAD) or control diet from embryonic day 14 up to 8 weeks of life (Fig. 5d). As expected, Aldefluor assays on VAD mice showed a reduction of RALDH ${ }^{+}$DCs compared with both $Z f p 36^{f / / f l}$ and $Z f p 36^{\Delta D C}$ mice on control diets (Fig. 5e). The frequency of splenic Tregs was only marginally affected by the VAD diet. In contrast, we observed a decrease in the proportion of SI LP Tregs in VAD Zfp $36^{\triangle D C}$ mice. Importantly, in these conditions, the frequency of $\mathrm{Foxp}^{+}$cells among $\mathrm{CD}^{+}$ $\mathrm{T}$ cells was comparable in $Z f p 36^{f / f l}$ and $Z f p 36^{\Delta \mathrm{DC}}$ mice, indicating that increased RALDH activity in TTP-deficient intestinal DCs controls local Treg expansion (Fig. 5f).

There are three different RALDHs in mice (RALDH1, RALDH2, and RALDH3 encoded by Aldh1a1, Aldh1a2, and Aldh1a3, respectively). We therefore quantified their mRNA expression in FACS-sorted SI LP DCs from Zfp $36^{\Delta \mathrm{DC}}$ mice. As a control, we sorted all $\mathrm{CD}_{11 \mathrm{c}^{-}}$cells. As compared with Zfp36 fl/fl, Zfp36 mRNA expression was strongly decreased in $\mathrm{CD}_{11 \mathrm{c}^{+}}$cells, validating our strategy for conditional deletion of this gene in DCs. Consistent with previous results, ${ }^{11}$ the absence of TTP promoted the expression of $1123 a$ in these cells. Aldh1a1 expression was comparable in both groups, while Aldh1a3 was undetectable. In contrast, we observed increased Aldh1a2 expression in Zfp36deficient SI LP DCs (Fig. 5g). We identified several putative ARE sequences in the $3^{\prime}$ UTR of the mRNA coding for RALDH2, suggesting that it could be directly regulated by TTP. To define whether Aldh1a2 is a bona fide TTP target, we performed in vitro experiments with bone marrow (BM) derived-DCs. After a short incubation with LPS to induce TTP expression, we evaluated Aldh1a2 mRNA half-life in WT and Zfp36-deficient cells by treating the cells with actinomycin D and SB203580, the latter used to abrogate the inhibitory action of p38 MAPK on TTP activation. ${ }^{7}$ As shown in Fig. 5h, Aldh1a2 mRNA stability was strongly increased in Zfp36-deficient BMDCs. Next, to assess if the putative ARE sequences found in the $3^{\prime} U T R$ of the mRNA coding for RALDH2 were sufficient to promote mRNA destabilization by TTP, we used a bidirectional reporter system ${ }^{23}$ in which we inserted either the full-length $3^{\prime}$ UTR sequence of Aldh1a2, or a truncated version lacking ARE motifs (Fig. 5i). Plasmids containing a synthetic (AUUU)8 ARE motif (AU8) or no ARE (AU0) were used as controls.
We evaluated the effect of TTP in co-transfection experiments in HEK293T cells. As expected, we observed a clear effect of TTP on the ratio between AU8 and AU0 reporter activities as compared with a control plasmid (expressing BOIP, encoded by Ccdc89) (Fig. 5j). Similar conclusions were reached for the construct containing Aldh1a2 3'UTR, with and without ARE motifs. Finally, to test the capacity of TTP to directly bind Aldh1a2 ARE, we performed an electrophoretic mobility supershift assay. We incubated the Aldh1a2 ARE probe with extracts from TTP-Flag expressing-HEK293T cells. Addition of a-Flag antibody resulted in a supershift that was not observed with BOIP-Flag or with an antiV5 antibody, demonstrating that TTP physically interacts with the Aldh $1 a 2$ mRNA ARE in this in vitro setting (Fig. 5k). Taken together, these results indicate that TTP directly regulates Aldh1a2 mRNA stability in intestinal DCs, and that this mechanism controls local Treg homeostasis.

\section{DISCUSSION}

Intestinal homeostasis relies on a coordinated set of innate and adaptive responses that calibrates responses against commensals and food-borne antigens. ${ }^{24}$ Activation of innate immune cells by the constant sensing of microbiota-derived signals has to be tightly regulated to avoid local inflammatory pathology. TTP represents a major mediator of dynamic posttranscriptional regulation. ${ }^{25}$ In immune and stromal cells, TTP regulates the expression of multiple inflammatory cytokines. ${ }^{26}$ It is therefore not surprising that $Z \mathrm{fp} 36^{-/-}$mice, which spontaneously develop a multiorgan inflammatory syndrome, also exhibit high levels of expression of inflammatory markers in both small and large intestines. We observed constitutive expression of TTP in intestinal DC and macrophage subpopulations. Intriguingly, TTP deficiency in $\mathrm{CD}_{11 \mathrm{c}^{+}}$cells or in myeloid cells was not sufficient to elicit increased expression of inflammatory markers in the gut. We reached similar conclusions in preliminary experiments when we targeted intestinal epithelial cells using Villin ${ }^{C r e} Z f p 36^{f / f l}$ mice. These results suggest that TTP deficiency in multiple cellular compartments could be required to drive dysregulated cytokine production. However, heightened expression of inflammatory cytokines in $\mathrm{Zfp}^{-1-}$ mice was not associated with any signs of intestinal pathology.

Tregs play a dominant role in the control of intestinal homeostasis, and the gut-associated lymphoid tissue represents a privileged site for the peripheral induction of Treg cells in response to oral antigens. ${ }^{27,28}$ This is related to the capacity of $\mathrm{CD}_{103^{+}}$DCs to produce RA. ${ }^{28,29}$ Although we did not demonstrate the heightened capacity of isolated intestinal TTP-deficient DCs to induce Treg development in in vitro assays, we show that TTP directly regulates RALDH2 in these cells, thereby influencing local Treg homeostasis. Of note, RA supplementation attenuates ileitis in $\operatorname{Tn}^{A A R E}$ mice by restoring the balance between Th17 and Treg cells. $^{30}$ Hence, we propose that in the absence of TTP, dysregulated production of inflammatory cytokines in the gut is balanced by enhanced Treg development and function. However, we did not formally demonstrate the role of the RALDH2-Treg axis in promoting intestinal homeostasis in TTP-deficient animals. Other regulatory pathways could contribute to the local control of inflammation in TTP-deficient animals. For instance, TTP was shown to target IL-10, a cytokine that plays a major role in the maintenance of intestinal homeostasis. ${ }^{31} \mathrm{IL}-10$ expression is inhibited in BM-derived macrophages from mice with the Zfp $36^{a a}$ gain-of-function mutation. However, the in vivo effects of this TTP mutation were uniformly anti-inflammatory, suggesting that this mechanism is not dominant. ${ }^{32}$ As TTP directly controls IL-22 production, this could also promote local mucosal integrity and homeostasis. ${ }^{33}$ Furthermore, a recent report indicated that deletion of Zfp36 in intestinal epithelial cells decreased the severity of dextran-sodium sulfate-induced colitis, possibly by 


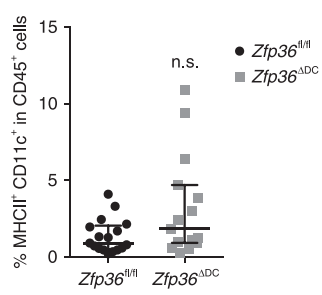

d

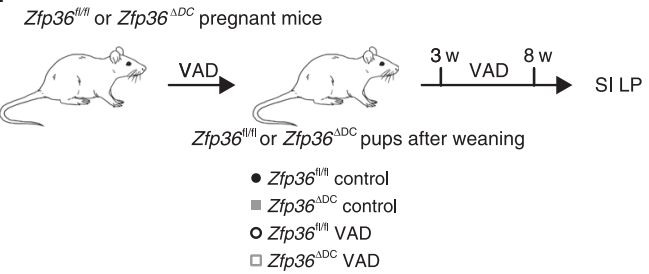

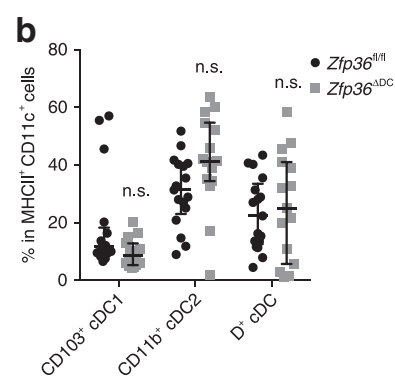

e
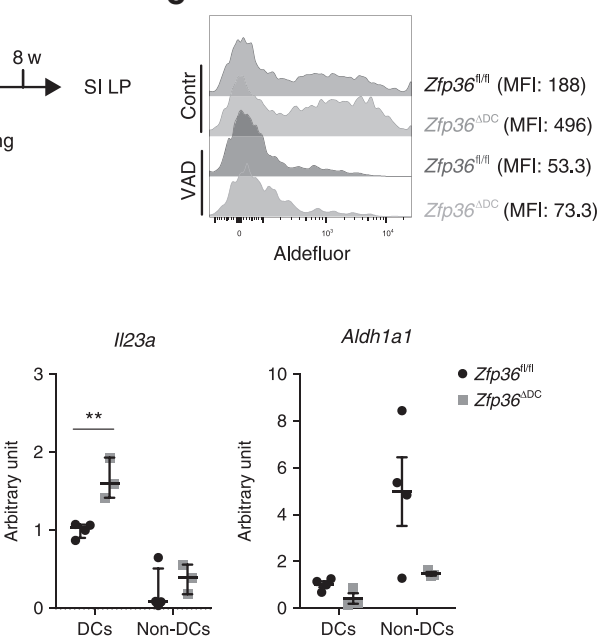

C

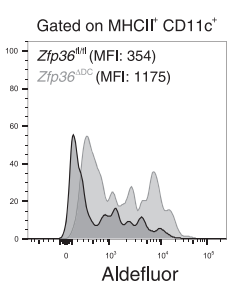

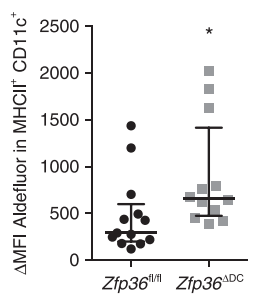

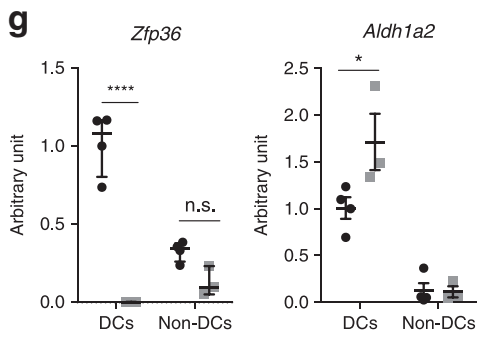

i

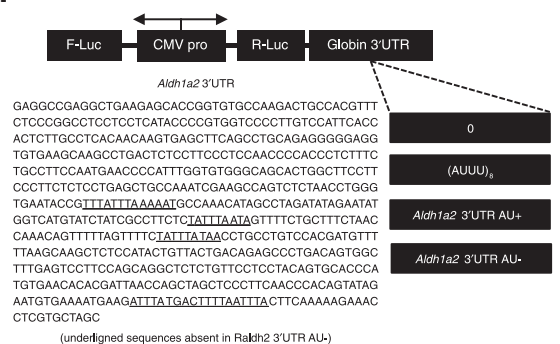

j

$$
\text { - BOIP-Flag }
$$

口 TTP-Flag

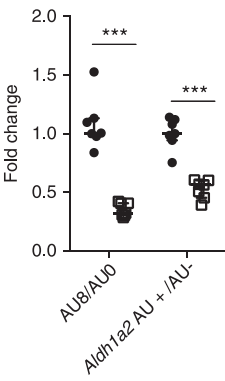

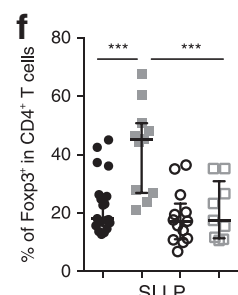
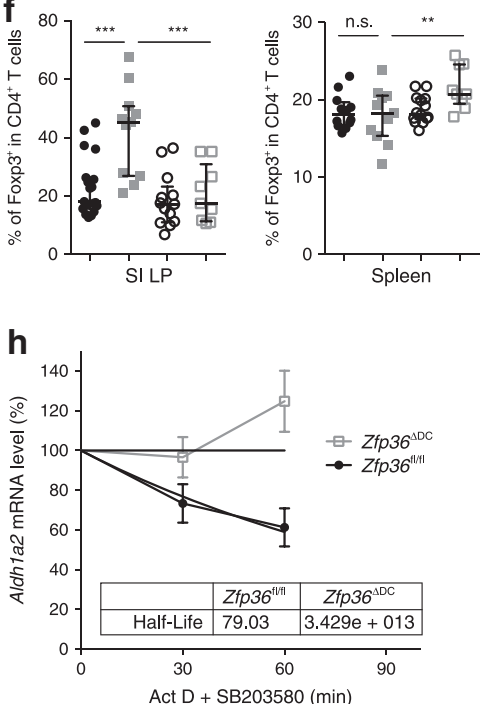

k

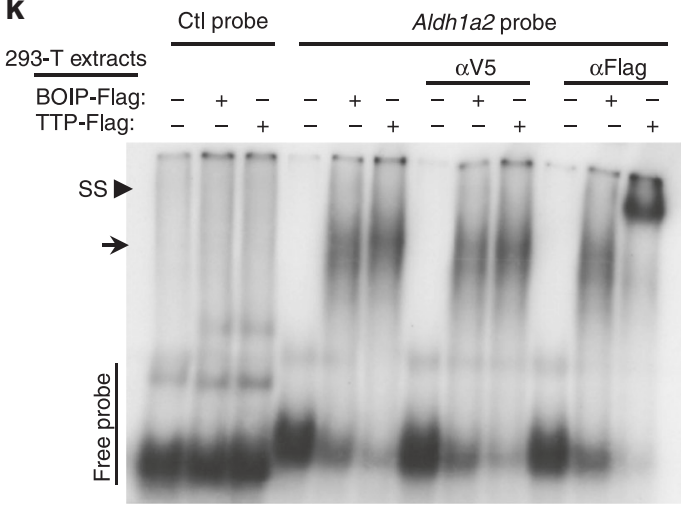

Fig. 5 Aldh1a2 mRNA is a direct target of TTP. a, b Flow cytometry results of DC subsets in SI LP of Zfp $36^{\mathrm{fl} / \mathrm{fl}}$ and $Z f p 36^{\Delta D C}$ mice. a Proportion of conventional dendritic cells $\left(\mathrm{MHCII}^{+} \mathrm{CD} 11 \mathrm{c}^{+}\right)$among live $\mathrm{CD}^{+} 5^{+}$cells $(n=15-17$ mice/group, pooled from three independent experiments). b Proportion of CDC subsets of the SI LP among CDCs ( $n=15-16$ mice/group, pooled from three independent experiments). c Aldefluor assay assessing RALDH activity in $\mathrm{MHCII}^{+} \mathrm{CD} 11 \mathrm{c}^{+}$cells of the SI LP by flow cytometry. Representative histogram (left) and $\triangle \mathrm{MFI}$ (MFI sample-(MFI sample+DEAB), see "Methods") (right) in the SI LP of $Z f p 36^{\mathrm{fl} / f f}$ and $Z f p 36^{\Delta D C}$ mice ( $n=12$ mice/group, pooled from three independent experiments). d Pregnant $Z f p 36^{\mathrm{f} / \mathrm{f}}$ or $Z f p 36^{\Delta D C}$ mice were fed with a vitamin A-deficient diet (VAD) that was maintained in the pups after weaning until sacrifice at 8 weeks of age, followed by flow cytometry experiments $(\mathbf{e}, \mathbf{f})$. Control mice were fed with a normal diet. e Representative Aldefluor in $\mathrm{MHCII}^{+} \mathrm{CD} 11 \mathrm{c}^{+}$cells in SI LP. f Proportion of Treg cells in SI LP and spleen ( $n=9-13$ mice/group, pooled from three independent experiments).

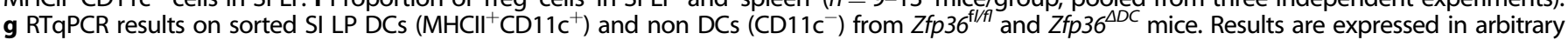
units, normalized against Rp/32 and Ubiquitin, and relative to the WT DCs group. Results from three independent experiments in which each dot originates from a pool of six mice. Statistical significance $\left({ }^{*} P<0.05,{ }^{* *} P<0.01\right)$ was assessed by two-way ANOVA test. $\mathbf{h}$ Half-life (in min) of Aldh1a2 mRNA in BMDCs from Zfp36 $6^{\mathrm{fl} / f l}$ or $Z f p 36^{\Delta D C}$ mice treated with LPS for $2 \mathrm{~h}$ prior to Actinomycin D $(10 \mu \mathrm{g} / \mu \mathrm{l})$ and SB203580 $(1 \mu \mathrm{M})$ treatment for the indicated time. mRNA was quantified by RTqPCR and normalized by mRNA levels at $t_{0}(n=3-4)$. i Schematic representation of the dual reporter plasmids generated to assess the role of the ARE elements: 3'UTR of Aldh1a2 mRNA with (AU+) or without (AU-) ARE was inserted behind the sequence coding for Renilla luciferase (R-Luc). Plasmids containing the WT Globin 3'UTR (AU0 = no ARE) or Globin 3'UTR with AU-rich insertion $(\mathrm{AUUU})_{8}(\mathrm{AU} 8=$ canonical ARE sequence) were used as control. The plasmids also contain the sequence coding for the Firefly luciferase (F-Luc) as a transfection efficiency control. Both luciferases are under the control of a bidirectional CMV promoter. $\mathbf{j}$ Fold change between the Rluc/Fluc ratio of $\mathrm{AU}+$ and $\mathrm{AU}$ - luciferase signals from dual reporter plasmids co-transfected with TTP-Flag (or BOIP-Flag as control) in HEK293T cells ( $n=7$ experiments). k Electrophoretic mobility supershift assay. Extracts from HEK293T cells transiently transfected with TTP-Flag or BOIP-flag were incubated with a ${ }^{32} \mathrm{P}$-labeled probe of Aldh1a2 ARE of a CTRL probe and either no antibody, an $\alpha$-V5 or an $\alpha$-Flag antibody before migration. Representative of three independent experiments. Statistical significance (ns nonsignificant, ${ }^{*} P<0.05,{ }^{* *} P<0.01,{ }^{* * *} P<0.001,{ }^{* * * *} P<0.0001$ ) was assessed by Mann-Whitney (a, $\mathbf{c})$ or by two-way ANOVA test $(\mathbf{b}, \mathbf{f}, \mathbf{g}, \mathbf{j})$. 
targeting Nos2. ${ }^{34}$ Altogether, these observations could account for the clear phenotypic differences between $\mathrm{Zfp} 6^{-/-}$and $T f^{\triangle A R E}$ mice.

Microbiota-derived signals set the immunological tone of tissues, both locally and systemically. ${ }^{24}$ Hence, the contribution of altered gut immune homeostasis to the pathogenesis of rheumatic diseases is an area of intense research. ${ }^{35}$ In particular, there is a clear connection between joint symptoms and gut inflammation in SpA. Up to $10 \%$ of AS patients also develop Crohn's disease during the course of the disease, and it is considered that nearly $50 \%$ of patients have subclinical gut inflammation. ${ }^{36}$ Of note, in line with the present observations in Zfp $36^{-1-}$ mice, ileal inflammation of AS patients is characterized by normal IL-17 levels despite increase in IL-23 expression. ${ }^{37}$ Strikingly, this immunological signature was also associated with expansion of IL-10-producing Tregs. ${ }^{38}$ Several studies reported alterations of the gut microbiota composition in patients with AS or psoriatic arthritis. ${ }^{16,39-41}$ Although $Z f p 36^{-/-}$mice did not display clear dysbiosis, we observed discrete alterations that are compatible to the ones described in AS patients. ${ }^{16}$ It is not clear, however, whether these perturbations are the consequence or the cause of inflammation. Nevertheless, we show that modulation of the gut microbiota with broad-spectrum antibiotics consistently reduced intestinal inflammatory markers as well as systemic, joint and skin inflammation. We cannot exclude that long-term oral antibiotic treatment also affects skin microbiota. Indeed, we previously demonstrated that invalidation of Zfp36 in epidermal cells was sufficient to trigger skin and also joint inflammation. ${ }^{15}$

Our results are consistent with the observation that raising HLAB27 transgenic rats in a germ-free environment prevents intestinal inflammation and reduces axial arthritis. ${ }^{42}$ Together with the observation that $Z \mathrm{fp} 36^{-/-}$mice bred in conventional housing develop more severe inflammation, ${ }^{15}$ these experiments support the notion that microbial-derived signals have an exacerbating effect on SpA pathological features. The cellular and molecular mechanisms at play remain ill-defined, but the present study indicates that $Z f p 36^{-1-}$ mice might represent an interesting preclinical model to address these mechanistic questions. It also provides a good rationale to evaluate the regulation of AREmediated RNA degradation in these patients.

In conclusion, our results begin to decipher the complex role of TTP in intestinal immune homeostasis, and provide a framework to study the interconnections between gut, skin, and joint inflammation in the context of SpA.

\section{MATERIALS AND METHODS}

Mice

Zfp36-deficient mice (Zfp36 $\left.6^{-1-}\right)$, LoxP-flanked Zfp36 mice $\left(Z f p 36^{\text {flox/flox }}\right)$ and Zfp36-V5 knock-in mice on a C57BL/6 background were previously described. ${ }^{10,19,20}$ CD11c-Cre (B6.Cg-Tg (Itgax-Cre)1-1Reiz), LysM-Cre (B6.129P2-Lyz2 ${ }^{\text {tm1(cre)lfo })}$ mice on a C57BL/6 background were purchased from The Jackson Laboratory. OTII Foxp3-GFP mice were generated by crossing OTII mice (B6.Cg$\mathrm{Tg}$ (TcraTcrb) $425 \mathrm{Cbn} / \mathrm{J}$ mice, purchased from The Jackson Laboratory) with Foxp3-DTR-GFP mice (B6.129(Cg)-Foxp3 ${ }^{\text {tm3(DTR/GFP)Ayr }}$ mice), which were kindly provided by Adrian Liston (KU Leuven) with permission from Alexander Rudensky (Howard Hughes Medical Institute and Immunology Program, Sloan-Kettering Institute, New York, NY). For antibiotic treatment, mice were treated for the indicated times with Vancomycin $500 \mathrm{mg} / \mathrm{l}$, Ampicillin $1 \mathrm{~g} / \mathrm{l}$, Neomycin $1 \mathrm{~g} / \mathrm{l}$ (Alfa Aesar), and Streptomycin $1 \mathrm{~g} / \mathrm{l}$ (PanReac Applichem) ad libitum in their drinking water. When indicated, pregnant females were fed VAD (SAFE) or sufficient diets from day 14 of gestation, and then kept on the same diet until weaning of the litter. ${ }^{43}$ Upon weaning, mothers were all returned to classical diets, while the weaned younglings were kept on the same special diet until 8 weeks of age, when the experiments were performed. All experiments were performed using littermates as controls. Male and female mice were used for the experiments. In each experiment, mice were age- and sex-matched. All mice were bred and maintained in a specific pathogen-free animal facility. All animal studies were approved by the institutional Animal Care and local committee for animal welfare of the BIOPOLE ULB CHARLEROI.

Flow cytometry analysis

Cells from spleen, MLN, LP, and skin were obtained as described in refs. ${ }^{15,43}$ Cells were resuspended in RPMI-1640 medium containing $10 \%$ FBS. Single-cell suspensions were incubated with anti-FcyllI/II receptor antibody and stained with fluorochromeconjugated mAb for phenotype analysis. LIVE/DEAD ${ }^{\mathrm{TM}}$ Fixable Near-IR dye (ThermoFischer) was used to exclude dead cells. Antibodies to TCRß-PerCP-Cy5.5/FITC (H57-597), CD4-PE-Cy7/ BV605 (RM4-5), CD8a-AF700 (53-6.7), Foxp3-APC/FITC (FJK-16s), RORyt-AF647 (Q31-378), GATA3-PE (L50-823), Tbet-PE (eBio4B10), IL-10-BV421 (JES5-16E3), IL-17A-APC/PE (TC11-18H10), MHCII (I-A/ E)-PE-Cy7/V500/FITC (M5/114.15.2), CD11c-PerCP-Cy5.5/PE-Cy7 (HL3), CD11b-PE/AF700 (M1/70), CD103-APC/BV421 (2E7/M290), Va2 TCR-PE (B20.1), VB5 TCR-biotin (MR9-4), V5 Tag-AF647, CD326PE-Cy7 (G8.8), CD45-FITC/AF700/BV510 (30-F11), Ly6G-PerCP-Cy5.5 (1A8), TCRy $\delta$-PerCP-eFluor710 (eBio-GL3), CD127-PE-Cy7 (SB/199), CD3- (145-2C11), CD19- (1D3), B220- (RA3-6B2), Gr1-APC-Cy7 (RB68C5), CD90.2-AF700 (30-H12), Ly6C-BV421/BV605 (AL-21), CD3BV421/BV711 (B3B4/145-2C11), Streptavidin-PE-Cy7/PerCP-Cy5.5 were purchased from Becton Dickinson, Biolegend, eBioscience, and Invitrogen (see references in Table S2). For cytokine staining, cells were incubated with PMA $(25 \mathrm{ng} / \mathrm{ml})$, ionomycin $(500 \mathrm{ng} / \mathrm{ml})$, and Brefeldin A $(20 \mu \mathrm{g} / \mathrm{ml})$ for $2 \mathrm{~h}$ (skin) or with PMA $(50 \mathrm{ng} / \mathrm{ml})$, ionomycin $(1 \mu \mathrm{g} / \mathrm{ml})$, and Brefeldin A $(20 \mu \mathrm{g} / \mathrm{ml})$ for $3 \mathrm{~h}(\mathrm{SI} L P)$ before staining. Intracellular staining was performed using Foxp3/ Transcription Factor Staining Buffer kit or Intracellular Fixation \& Permeabilization kit (eBioscience) for transcription factor and cytokine analysis, respectively. Concomitant staining for Fopx3 and IL-10 was performed using Foxp3/Transcription Factor Staining Buffer kit (eBioscience). Data were collected on a BD LSRII Fortessa or FACSCanto ${ }^{\mathrm{TM}}$ II (BD Bioscience) and analyzed with FlowJo v10 software.

\section{Arthritis scoring}

The paws were monitored for clinical symptoms every week from 6 weeks until 3 months of age, and then every 2 weeks until sacrifice. Disease severity scoring system: $0=$ normal; $1=$ swelling of one toe; 2 =swelling of more than one toe in the same paw; 3 = swelling of the entire paw or several toes in different paws; $4=$ ankylosed paw.

\section{Oral tolerance model}

Single-cell suspension of spleen and lymph nodes were obtained from OTII Foxp3-GFP mice and enriched in $\mathrm{CD}^{+} \mathrm{T}$ cells by positive selection on a MACS column (Miltenyi). Cells were then labeled with fluorescent dye conjugated monoclonal anti-TCR $\beta$ and antiCD4 antibodies and sorted by flow cytometry using FACSAria III (BD Bioscience) as $\mathrm{TCR}^{+} \mathrm{CD}^{+} \mathrm{GFP}^{-}$cells up to $>95 \%$ purity. Purified naive T cells from OTII Foxp3-GFP mice were adoptively transferred into $Z f p 36^{\text {flox/flox }}$ or $Z f p 36^{\Delta D C}$ mice. Each mouse was injected i.v. with at least $1.5 \times 10^{6}$ naive $T$ cells in phosphatebuffered saline (PBS). Mice were then fed a $1.5 \%$ OVA solution (Sigma-Aldrich) in drinking water for 6 days. On day 6, cell suspensions from spleen, MLN, and SI LP were prepared for flow cytometry analysis.

\section{Toxoplasma gondii infection}

ME-49 type II strain of Toxoplasma gondii was kindly provided by Dr De Craye (ISP, Belgium) and was used for the production of tissue cysts in C57BL/6 mice. For acute T.gondii infection, cysts 
collected from the brains of chronically infected mice were counted, and mice were fed ten T.gondii cysts in PBS solution per oral gavage $(200 \mu \mathrm{l} /$ mouse). Mice were sacrificed at day 7 post infection for analysis.

\section{Histology}

Mouse tissues were fixed in $4 \%$ paraformaldehyde and directly paraffin embedded. Sections $(6 \mu \mathrm{m})$ were stained with hematoxylin-eosin. Villus length and crypt depth were measured using the Leica Image Viewer Software or NanoZoomer Digital Pathology software; a mean of 10 measures was calculated for each parameter per sample.

\section{Aldefluor assay}

An ALDEFLUOR ${ }^{\text {TM }}$ assay kit (STEMCELL Technologies) was used according to the manufacturer's instruction. Briefly, cells were stained with LIVE/DEAD ${ }^{\mathrm{TM}}$ Fixable Near-IR dye (APC-Cy7), MHCII (IA/E)-PE-Cy7 (M5/114.15.2), and CD11C-PerCP-Cy5.5 (HL3), and then incubated with ALDEFLUOR ${ }^{\mathrm{TM}}$ activated reagent with or without DEAB inhibitor for $30 \mathrm{~min}$ at $37^{\circ} \mathrm{C}$ in the dark. Cells were washed and suspended in ALDEFLUOR ${ }^{\mathrm{TM}}$ buffer for rapid flow cytometry analysis. Gating strategies used were as follow: for each sample, the gate for Aldefluor ${ }^{+}$cells was placed according to the same sample $+D E A B$, so that the Aldefluor ${ }^{+}$cells in the sample $+D E A B$ represented $<1 \%$ of parent population. (R)ALDH activity was assessed by the MFI of Aldefluor in DCs. To correct for background fluorescence, the MFI for Aldefluor in the cell sample incubated with DEAB was subtracted from the MFI for Aldefluor in the same sample incubated without inhibitor. The results are presented as $\left.\Delta \mathrm{MFI}=\mathrm{MFI}_{\text {(sample) }}-\mathrm{MFI}_{\text {(sample }}+\mathrm{DEAB}\right)$.

Sorting of LP dendritic cells

LP cells were isolated and stained as described above, then sorted on BD FACSAria III (SI LP DCs = live MHCII ${ }^{+}$CD $11 c^{\text {hi }}$ and non $\mathrm{DCs}=$ live $\mathrm{CD} 11 \mathrm{c}^{-}$). The collected cells were resuspended in TRIzol reagent (Thermo Fisher) to proceed to RNA extraction.

\section{Gene expression}

Total RNA was extracted with NucleoSpin RNA (Macherey) and reverse-transcribed with the RevertAid RT Reverse Transcription kit (Thermo Fisher). cDNA was amplified using SYBR green or TaqMan probes. Primer sequences are available in Table S3. Half-life of mRNAs was determined using nonlinear regression (one phase decay) in GraphPad Prism.

\section{Cytokines production}

The concentration of LCN2 was determined in serum or feces by ELISA (R\&D system). Feces were first homogenized in PBS using MagNA Lyser green beads (Roche). Supernatants were then collected to proceed to ELISA experiments.

\section{Gut microbiota analysis}

Genomic DNA was extracted from feces of littermate mice separately housed according to their genotype after weaning using a QIAamp DNA Stool Mini Kit (Qiagen), including a beadbeating step with zirconia beads (BioSpec). Absolute quantification of the total bacteria levels was performed using qPCR (primers presented in Table S4). The samples were PCR-enriched for the V5-V6 region of the 16S rRNA gene, and then underwent a library tailing PCR (primers in Table S4). The amplicons were purified, quantified, and sequenced using an Illumina MiSeq to produce $2 \times 300$ bp sequencing products. Initial quality-filtering of the reads was conducted with the Illumina Software, yielding an average of 97,296 pass-filter reads per sample. Quality scores were visualized, and reads were trimmed to $220 \mathrm{bp}$ (R1) and $200 \mathrm{bp}$ (R2). The reads were merged with the mergeIllumina pairs application. ${ }^{44}$ All samples were subsampled to 33,000 sequences per sample using Mothur $1.32 .1 .^{45}$ The
UPARSE pipeline implemented in USEARCH was used to further process the sequences. ASVs were identified using UNOISE3. ${ }^{46}$ Taxonomic prediction was performed using the nbc_tax function, an implementation of the RDP Naive Bayesian Classifier algorithm. ${ }^{47}$ The phylotypes were computed as percent proportions based on the total number of sequences in each sample. Beta-diversity indexes were calculated using QIIME. ${ }^{48}$ PCoA plot of the beta-diversity indexes were obtained using EMPeror. ${ }^{49}$

\section{Cell culture}

HEK293T cells (ATCC) were maintained in DMEM medium (Gibco) containing 10\% FBS, $50 \mathrm{U} / \mathrm{ml}$ Penicillin, $50 \mu \mathrm{g} / \mathrm{ml}$ Streptamycin (Pen Strep, Gibco), and $1 \mathrm{mM}$ sodium pyruvate (Gibco). Transfections were performed using the calcium phosphate method of transfection. Plasmid DNA was added to a $\mathrm{CaCl}_{2}$ solution, then added dropwise into a Hepes-buffered phosphate solution. This DNA solution is then incubated at room temperature for $30 \mathrm{~min}$ before being spotted on the cell culture. Cells' medium was changed prior to adding the DNA and after incubation of the cells overnight with the DNA mixture. Cells were harvested on day 3 for experiments. BMDC were differentiated from fresh BM using GM-CSF as follows: Cells obtained from the BM of $Z \mathrm{fp} 36^{+/+}$and $Z \mathrm{fp} 36^{-/-}$mice were plated at $0.5 \times 10^{6}$ cells $/ \mathrm{ml}$ in Petri dishes $(10 \mathrm{ml} /$ dish) in RPMI1640 medium (Lonza) containing 10\% HyClone FBS (LPS-free), $50 \mathrm{U} / \mathrm{ml}$ Penicillin, $50 \mu \mathrm{g} / \mathrm{ml}$ Streptamycin (Pen Strep, Gibco), 1 $\mathrm{mM}$ sodium pyruvate (Gibco) and nonessential amino acids (Gibco, dilution 100) and supplemented with GM-CSF $(20 \mathrm{ng} / \mathrm{ml}$, RnD Systems). Cells were fed at day 3 and 6 of culture with fresh medium containing GM-CSF $(20 \mathrm{ng} / \mathrm{ml})$. At day 7 , cells were seeded $\left(1.5\right.$ to $\left.2 \times 10^{6} \mathrm{cell} / \mathrm{ml}\right)$ for experiments the next day. For half-life experiments, at day 8 , BMDCs were stimulated with LPS $(100 \mathrm{ng} / \mathrm{ml}$, Sigma) for $2 \mathrm{~h}$ before treatment with Actinomycin D $(10 \mu \mathrm{g} / \mathrm{ml}$, Sigma) and the P38 inhibitor SB203580 $(1 \mu \mathrm{M}$, Calbiochem) for the indicated times.

Dual reporter plasmids

The WT 3'UTR of Aldh1a2 gene was amplified by RT-PCR using the primers $5^{\prime}$-agctagagcggccgcggatccgaggccgaggctgaagagca- $3^{\prime}$ and $5^{\prime}$-gctcgaagcggccgcgctagcacgaggtttcttttgaa- $3^{\prime}$, and then cloned into the Notl site of the $\mathrm{AU}_{0}$ plasmid from Barreau et al., ${ }^{23}$ using a ligation independent cloning method. For RALDH2 $\triangle A R E$, a DNA fragment containing the $3^{\prime} U T R$ of RALDH without the putative ARE motifs was synthesized (Integrated DNA Technologies), and then amplified by PCR before cloning in the $A U_{0}$ plasmid.

Electrophoretic mobility supershift assay

Analysis of possible Aldh1a2 mRNA-TTP interactions by the EMSA supershift assay was performed as described in ref. ${ }^{50}$ with the following modifications. Cell extracts from HEK293T cells transfected with TTP-Flag of BOIP-Flag were incubated with ${ }^{32} \mathrm{P}$-labeled RNA probe, followed by the addition of anti-V5 or anti-Flag antibody prior to sample loading on $5 \%$ polyacrylamide nondenaturing gels containing $6 \%$ glycerol and $0.5 \mathrm{X}$ TBE at $7.5 \mathrm{~mA}$ for $16 \mathrm{~h}$ at $4^{\circ} \mathrm{C}$.

Statistical analysis

The results are expressed as median \pm interquartile range. The statistical significance was assessed using the two-tail Mann-Whitney test, the Kruskal-Wallis test with Dunn correction or the two-way ANOVA test with Sidak correction (GraphPad Prism 6.0). Results were considered significant for ${ }^{*} P<0.05,{ }^{* *} P<0.01,{ }^{* * *} P<0.001$, and ${ }^{* * *} P<0.0001$. For the gut microbiota sequencing results, $p$ values were corrected using the Benjamini-Hochberg correction for multiple testing ( $q$ value) and the significance threshold was placed at $q$ value $<0.1$. 


\section{DATA AVAILABILITY}

Raw data generated during the analysis of the gut microbiota composition can be accessed on SRA (SRA accession: PRJNA558549).

\section{ACKNOWLEDGEMENTS}

This study was supported by the Fonds National de la Recherche Scientifique (FRSFNRS, Belgium), the WELBIO, by the European Regional Development Fund (ERDF) of the Walloon Region (Wallonia-Biomed portfolio, 411132-957270), and the "Actions de Recherches Concertées" (AV.12/17). This work was supported in part by the Intramural Research Program of the NIH, NIEHS (PJB). We thank Jacques and Alexandre Delen for their support. SG is a senior research associate of the FRS-FNRS. $\mathrm{CL}$ was supported by PhD fellowship from the FRS-FNRS and by the Fonds ERASME. BdT was supported by a PhD fellowship of the Belgian Fonds pour la Recherche en Industrie et Agriculture (FRIA). We thank Bouazza Es Saadi and Rose-Marie Goebbels for technical assistance. Histology was performed by the Center for Microscopy and Molecular Imaging (CMMI), which is supported by the Walloon Region and the ERDF (Wallonia-Biomed portfolio, 411132-957270).

\section{AUTHOR CONTRIBUTIONS}

$\mathrm{CL}$ and BdT conducted most of the experiments. LVM, AA, MM, and $\mathrm{HL}$ contributed to some experiments. MN, ST, JS, AK, RS, and ND provided technical help for the experiments. LBB performed microbiota analysis. $\mathrm{CL}$ and BdT analyzed the data and prepared the figures. $\mathrm{LBB}, \mathrm{PJB}$, and VK provided input for research design and interpretation. WH provided critical reagents. SG, GO, and CG supervised the work and wrote the manuscript. All authors were involved in critically revising the manuscript for important intellectual content. All authors had full access to the data and approved the manuscript before it was submitted by the corresponding author.

\section{ADDITIONAL INFORMATION}

The online version of this article (https://doi.org/10.1038/s41385-020-0302-x) contains supplementary material, which is available to authorized users.

Competing interests: The authors declare no competing interests.

Publisher's note Springer Nature remains neutral with regard to jurisdictional claims in published maps and institutional affiliations.

\section{REFERENCES}

1. Hao, S. \& Baltimore, D. The stability of mRNA influences the temporal order of the induction of genes encoding inflammatory molecules. Nat. Immunol. 10, 281-288 (2009).

2. Kontoyiannis, D., Pasparakis, M., Pizarro, T. T., Cominelli, F. \& Kollias, G. Impaired on/off regulation of TNF biosynthesis in mice lacking TNF AU-rich elements: implications for joint and gut-associated immunopathologies. Immunity 10, 387-398 (1999).

3. Schaubeck, M. et al. Dysbiotic gut microbiota causes transmissible Crohn's disease-like ileitis independent of failure in antimicrobial defence. Gut 1-13 https://doi.org/10.1136/gutjnl-2015-309333 (2015).

4. Roulis, M. et al. Host and microbiota interactions are critical for development of murine Crohn's-like ileitis. Mucosal. Immunol. 9, 1-11 (2015).

5. Clement, S. L., Scheckel, C., Stoecklin, G. \& Lykke-Andersen, J. Phosphorylation of tristetraprolin by MK2 impairs AU-rich element mRNA decay by preventing deadenylase recruitment. Mol. Cell. Biol. 31, 256-266 (2011).

6. Sandler, H., Kreth, J., Timmers, H. T. M. \& Stoecklin, G. Not1 mediates recruitment of the deadenylase Caf1 to mRNAs targeted for degradation by tristetraprolin. Nucleic Acids Res. 39, 4373-4386 (2011).

7. Kratochvill, F. et al. Tristetraprolin-driven regulatory circuit controls quality and timing of mRNA decay in inflammation. Mol. Syst. Biol. 7, 560 (2011).

8. Qian, X. et al. Posttranscriptional regulation of IL-23 expression by IFN-gamma through tristetraprolin. J. Immunol. 186, 6454-6464 (2011).

9. Carballo, E., Lai, W. S. \& Blackshear, P. J. Evidence that tristetraprolin is a physiological regulator of granulocyte-macrophage colony-stimulating factor messenger RNA deadenylation and stability. Blood 95, 1891-1899 (2000).

10. Taylor, G. A. et al. A pathogenetic role for TNF alpha in the syndrome of cachexia, arthritis, and autoimmunity resulting from tristetraprolin (TTP) deficiency. Immunity 4, 445-454 (1996).

11. Molle, C. et al. Tristetraprolin regulation of interleukin 23 mRNA stability prevents a spontaneous inflammatory disease. J. Exp. Med. 210, 1675-1684 (2013).

12. Gracey, E. et al. The ties that bind: skin, gut and spondyloarthritis. Curr. Opin. Rheumatol. 31, 62-69 (2019).
13. Sieper, J. \& Poddubnyy, D. Axial spondyloarthritis. Lancet 390, 73-84 (2017).

14. Brown, M. A., Kenna, T. \& Wordsworth, B. P. Genetics of ankylosing spondylitisinsights into pathogenesis. Nat. Publ. Gr. 12, 81-91 (2015).

15. Andrianne, M. et al. Tristetraprolin expression by keratinocytes controls local and systemic inflammation. JCl Insight 2, 1-16 (2017).

16. Costello, M. et al. Intestinal Dysbiosis in Ankylosing Spondylitis. Arthritis Rheumatol. 67, 686-691 (2015).

17. Błażejewski, A. J. et al. Microbiota normalization reveals that canonical caspase-1 activation exacerbates chemically induced intestinal inflammation. Cell Rep. 19, 2319-2330 (2017).

18. Tanoue, T., Atarashi, K. \& Honda, K. Development and maintenance of intestinal regulatory T cells. Nat. Rev. Immunol. 16, 295-309 (2016).

19. Zhang, X., Chen, X., Liu, Q., Zhang, S. \& Hu, W. Translation repression via modulation of the cytoplasmic poly(A)-binding protein in the inflammatory response. Elife 6, e27786 (2017).

20. Qiu, L.-Q., Stumpo, D. J. \& Blackshear, P. J. Myeloid-specific tristetraprolin deficiency in mice results in extreme lipopolysaccharide sensitivity in an otherwise minimal phenotype. J. Immunol. 188, 5150-5159 (2012).

21. Oldenhove, G. et al. Decrease of Foxp3+ Treg cell number and acquisition of effector cell phenotype during lethal infection. Immunity 31, 772-786 (2009).

22. Jaensson, E. et al. Small intestinal CD103+ dendritic cells display unique functional properties that are conserved between mice and humans. J. Exp. Med. 205, 2139-2149 (2008)

23. Barreau, C., Watrin, T., Beverley Osborne, H. \& Paillard, L. Protein expression is increased by a class III AU-rich element and tethered CUG-BP1. Biochem. Biophys. Res. Commun. 347, 723-730 (2006).

24. Belkaid, Y. \& Harrison, O. J. Homeostatic Immunity and the Microbiota. Immunity 46, 562-576 (2017).

25. Rabani, M. et al. High-resolution sequencing and modeling identifies distinct dynamic RNA regulatory strategies. Cell 1-13. https://doi.org/10.1016/j.cell.2014.11.015 (2014).

26. Fu, M. \& Blackshear, P. J. RNA-binding proteins in immune regulation: a focus on CCCH zinc finger proteins. Nat. Rev. Immunol. 17, 130-143 (2017).

27. Mucida, D. et al. Oral tolerance in the absence of naturally occurring Tregs. J. Clin. Investig. 115, 1923-1933 (2005).

28. Coombes, J. L. et al. A functionally specialized population of mucosal CD103+ DCs induces Foxp3+ regulatory $T$ cells via a TGF-beta and retinoic aciddependent mechanism. J. Exp. Med. 204, 1757-1764 (2007).

29. Esterházy, D. et al. Classical dendritic cells are required for dietary antigenmediated induction of peripheral T(reg) cells and tolerance. Nat. Immunol. 17, 545-555 (2016).

30. Collins, C. B. et al. Retinoic acid attenuates ileitis by restoring the balance between T-helper 17 and T regulatory cells. Gastroenterology 141, 1821-1831 (2011).

31. Stoecklin, G. et al. Genome-wide analysis identifies interleukin-10 mRNA as target of tristetraprolin. J. Biol. Chem. 283, 11689-11699 (2008).

32. O'Neil, J. D. et al. Gain-of-function mutation of tristetraprolin impairs negative feedback control of macrophages in vitro yet has overwhelmingly antiinflammatory consequences in vivo. Mol. Cell. Biol. 37, e00536-16 (2017).

33. Härdle, L. et al. Tristetraprolin regulation of interleukin-22 production. Sci. Rep. 5, 15112 (2015).

34. Eshelman, M. A. et al. Tristetraprolin targets Nos2 expression in the colonic epithelium. Sci. Rep. 9, 14413 (2019).

35. Van de Wiele, T., Van Praet, J. T., Marzorati, M., Drennan, M. B. \& Elewaut, D. How the microbiota shapes rheumatic diseases. Nat. Rev. Rheumatol. 12, 398-411 (2016).

36. Jacques, P., Van Praet, L., Carron, P., Van den Bosch, F. \& Elewaut, D. Pathophysiology and role of the gastrointestinal system in spondyloarthritides. Rheum. Dis. Clin. North Am. 38, 569-582 (2012).

37. Ciccia, F. et al. Overexpression of interleukin-23, but not interleukin-17, as an immunologic signature of subclinical intestinal inflammation in ankylosing spondylitis. Arthritis Rheum. 60, 955-965 (2009).

38. Ciccia, F. et al. Expansion of intestinal CD4+CD25high Treg cells in patients with ankylosing spondylitis: a putative role for interleukin-10 in preventing intestinal Th17 response. Arthritis Rheum. 62, 3625-3634 (2010).

39. Scher, J. U. et al. Decreased bacterial diversity characterizes the altered gut microbiota in patients with psoriatic arthritis, resembling dysbiosis in inflammatory bowel disease. Arthritis Rheumatol. 67, 128-139 (2015).

40. Wen, C. et al. Quantitative metagenomics reveals unique gut microbiome biomarkers in ankylosing spondylitis. Genome Biol. 18, 142 (2017).

41. Breban, M. et al. Faecal microbiota study reveals specific dysbiosis in spondyloarthritis. Ann. Rheum. Dis. 76, 1614-1622 (2017).

42. Taurog, J. D. et al. The germfree state prevents development of gut and joint inflammatory disease in HLA-B27 transgenic rats. J. Exp. Med. 180, 2359-2364 (1994).

43. Francois, V. et al. Intestinal immunopathology is associated with decreased CD73generated adenosine during lethal infection. Mucosal Immunol. 8, 773-784 (2015). 
The RNA-binding protein tristetraprolin regulates RALDH2 expression by... C La et al.

44. Eren, A. M., Vineis, J. H., Morrison, H. G. \& Sogin, M. L. A filtering method to generate high quality short reads using illumina paired-end technology. PLoS ONE 8, e66643 (2013).

45. Schloss, P. D. et al. Introducing mothur: open-source, platform-independent, community-supported software for describing and comparing microbial communities. Appl. Environ. Microbiol. 75, 7537-7541 (2009).

46. Edgar, R. C. UNOISE2: improved error-correction for Illumina $16 \mathrm{~S}$ and ITS amplicon sequencing. bioRxiv. https://doi.org/10.1101/081257 (2016).
47. Edgar, R. C. Accuracy of taxonomy prediction for $16 \mathrm{~S}$ rRNA and fungal ITS sequences. PeerJ 6, e4652 (2018).

48. Caporaso, J. G. et al. QIIME allows analysis of high-throughput community sequencing data. Nat. Methods 7, 335-336 (2010).

49. Vázquez-Baeza, Y., Pirrung, M., Gonzalez, A. \& Knight, R. EMPeror: a tool for visualizing high-throughput microbial community data. Gigascience 2, 16 (2013).

50. Gueydan, C. et al. Engagement of tumor necrosis factor mRNA by an endotoxininducible cytoplasmic protein. Mol. Med. 2, 479-488 (1996). 